\title{
MYC amplification at diagnosis drives therapy- induced hypermutation of recurrent glioma
}

Jiguang Wang ( $\sim$ jgwang@ust.hk)

The Hong Kong University of Science and Technology https://orcid.org/0000-0002-6923-4097

\section{Quanhua Mu}

The Hong Kong University of Science and Technology

\section{Ruichao Chai}

Beijing Neurosurgical Institute

Hanjie Liu

Beijing Neurosurgical Institute

\section{Yingxi Yang}

Division of Life Science and Department of Chemical and Biological Engineering, Hong Kong University of Science and Technology, Hong Kong

\section{Zheng Zhao}

Beijing Neurosurgical Institute

\section{Ming Hong Lui}

Division of Life Science and Department of Chemical and Biological Engineering, Hong Kong University of Science and Technology, Hong Kong

\section{Zhaoshi Bao}

Beijing Neurosurgical Institute

\section{Dong Song}

hong kong university of science and technology https://orcid.org/0000-0002-5213-5121

\section{Biaobin Jiang}

Division of Life Science and Department of Chemical and Biological Engineering, Hong Kong University of Science and Technology, Hong Kong

\section{Jason Sa}

Korea University https://orcid.org/0000-0002-3251-5004

\section{Hee Jin Cho}

Samsung Medical Center

\section{Yuzhou Chang}

Beijing Neurosurgical Institute

\section{Kaitlin Hao Yi Chan}

HKUST https://orcid.org/0000-0001-7363-2082

\section{Danson Shek Chun Loi}




\section{Sindy Sing Ting Tam}

HKUST

\section{Aden Ka Yin Chan}

CUHK

\section{Angela Wu}

Division of Life Science and Department of Chemical and Biological Engineering, Hong Kong University of Science and Technology, Hong Kong https://orcid.org/0000-0002-3531-4830

\section{Wai San Poon}

The Chinese University of Hong Kong

\section{H.K. Ng}

The Chinese University of Hong Kong

\section{Danny Chan}

Prince of Wales Hospital

\section{Antonio lavarone}

Columbia University https://orcid.org/0000-0002-0683-4634

\section{Do-Hyun Nam}

Samsung Medical Center

\section{Tao Jiang}

Beijing Neurosurgical Institute https://orcid.org/0000-0002-7008-6351

\section{Biological Sciences - Article}

Keywords: Clonal Evolution, Adult Diffuse Gliomas, Matched Initial-recurrent Tumor Pairs, Grade Progression, Loss-of-function Mutations, Cancer Evolution

Posted Date: January 5th, 2021

DOI: https://doi.org/10.21203/rs.3.rs-138020/v1

License: (c) (i) This work is licensed under a Creative Commons Attribution 4.0 International License. Read Full License 


\section{MYC amplification at diagnosis drives therapy-induced hypermutation}

\section{2 of recurrent glioma}

4 Quanhua $\mathrm{Mu}^{1, \#}$, Ruichao Chai ${ }^{2, \#}$, Hanjie $\mathrm{Liu}^{2}$, Yingxi Yang ${ }^{1}$, Zheng Zhao ${ }^{2}$, Ming Hong

5 Lui $^{1}$, Zhaoshi Bao ${ }^{1,2}$, Dong Song ${ }^{1}$, Biaobin Jiang ${ }^{1}$, Jason K. Sa ${ }^{4,5}$, Hee Jin Cho ${ }^{4}$, Yuzhou

$6 \mathrm{Chang}^{2}$, Kaitlin Hao Yi Chan ${ }^{3}$, Danson Shek Chun Loi ${ }^{3}$, Sindy Sing Ting Tam³ ${ }^{3}$ Aden Ka

7 Yin $\mathrm{Chan}^{7}$, Angela Ruohao $\mathrm{Wu}^{3}$, Wai Sang Poon ${ }^{6}$, Ho Keung Ng${ }^{7}$, Danny Tat Ming Chan ${ }^{6}$,

8 Antonio Iavarone ${ }^{8, *}$, Do-Hyun Nam ${ }^{4,9,10,11, *}$, Tao Jiang ${ }^{2,11, *}$, Jiguang Wang ${ }^{1,11, *}$

$10{ }^{1}$ Division of Life Science, Department of Chemical and Biological Engineering, Center of

11 Systems Biology and Human Health, State Key Laboratory of Molecular Neuroscience,

12 The Hong Kong University of Science and Technology, Hong Kong SAR, China.

$13 \quad{ }^{2}$ Beijing Neurosurgical Institute, Capital Medical University, Beijing, China.

$14{ }^{3}$ Division of Life Science, Department of Chemical and Biological Engineering, The Hong

15 Kong University of Science and Technology, Hong Kong SAR, China.

$16{ }^{4}$ Institute for Refractory Cancer Research, Samsung Medical Center, Seoul, South Korea.

$17{ }^{5}$ Department of Biomedical Sciences, Korea University College of Medicine, Seoul, Korea.

$18{ }^{6}$ CUHK Otto Wong Brain Tumour Centre, Department of Surgery, Prince of Wales

19 Hospital, The Chinese University of Hong Kong, Hong Kong SAR, China.

$20 \quad{ }^{7}$ Department of Anatomical and Cellular Pathology, Prince of Wales Hospital, The Chinese

21 University of Hong Kong, Hong Kong SAR, China.

$22{ }^{8}$ Institute for Cancer Genetics, Columbia University, New York, New York, USA. 
$23{ }^{9}$ Department of Neurosurgery, Samsung Medical Center, Sungkyunkwan University

24 School of Medicine, Seoul, Korea.

$25{ }^{10}$ Department of Health Science \& Technology, Samsung Advanced Institute for Health

26 Sciences \& Technology, Sungkyunkwan University School of Medicine, Seoul, Korea.

$27{ }^{11}$ Chinese Glioma Genome Atlas (CGGA) and Asian Glioma Genome Atlas (AGGA)

28 Research Networks.

29

30 \# These authors contributed equally

$31 *$ Emails:

32 igwang@ust.hk; ai2102@cumc.columbia.edu; nsnam@skku.edu; taojiang1964@163.com. 


\section{Abstract}

2 Clonal evolution drives cancer progression and therapeutic resistance ${ }^{1,2}$. Recent

3 longitudinal analyses revealed divergent clonal dynamics in adult diffuse gliomas $^{3-11}$.

4 However, the early genomic and epigenomic factors that steer post-treatment molecular

5 trajectories remain unknown. To track evolutionary predictors, we analyzed sequencing

6 and clinical data of matched initial-recurrent tumor pairs from 511 adult diffuse glioma

7 patients. Using machine learning we developed methods capable of predicting grade

8 progression and hypermutation from tumor characteristics at diagnosis. Strikingly, $M Y C$

9 copy number gain in initial tumors emerged as a key factor predicting development of

10 hypermutation under temozolomide (TMZ) treatment. The driving role of MYC in TMZ-

11 associated hypermutagenesis has been experimentally validated in a model of TMZ-

12 induced hypermutator using both patient-derived gliomaspheres and established glioma

13 cell lines. Subsequent studies showed that c-Myc binding to open chromatin and

14 transcriptionally active regions increases the vulnerability of genomic regions to TMZ-

15 induced mutagenesis. Consequently, MYC target genes, including the key mismatch repair

16 genes, develop loss-of-function mutations, thus triggering the hypermutation process. This

17 study reveals MYC as an early predictor of cancer evolution and provides a machine

18 learning platform for predicting cancer dynamics to improve patient management. 


\section{Main}

2 Malignant diffuse gliomas (WHO grade II to IV) constitute the most common primary

3 brain tumors in adults ${ }^{12}$. Despite surgery combined with radiotherapy plus alkylator

4 chemotherapy, aggressive gliomas inevitably recur. At relapse, a proportion of lower-grade

5 gliomas (LGG) remain grade II-III but a remarkable number of cases progress into grade

6 IV glioblastoma (GBM), leading to worse prognosis ${ }^{3}$. Molecular mechanisms that drive

7 this process remain elusive.

9 To characterize the evolutionary landscape of glioma under therapy, enormous efforts were

10 devoted to sequence cancer genomes at multiple time points ${ }^{3-11}$. The hypermutation (HM)

11 phenomenon was detected in temozolomide-treated samples in several independent cohorts,

12 highlighting the role of stress-induced mutagenesis in shaping adaptive glioma

13 evolution ${ }^{13,14}$. Our recent study revealed a mutually exclusive pattern between HM and an

14 alternative TMZ-resistant mechanism driven by $M G M T^{15}$. Moreover, group efforts of

15 Glioma Longitudinal AnalySiS (GLASS) consortium reconstructed molecular trajectories

16 of 222 individual cases, demonstrating a heterogeneous and largely stochastic pattern of

17 glioma evolution after treatment ${ }^{8}$. Yet, it is still unknown how the evolutionary routes were

18 determined and whether the early predictors of cancer evolution exist.

20 Here we analyzed longitudinal sequencing data from a large number of diffuse glioma

21 cases that cover all three molecular subtypes-IDH wildtype (IDHwt), IDH-mutant

22 without chromosome $1 \mathrm{p} / 19 \mathrm{q}$ co-deletion (IDHmut-noncodel), and IDH-mutant with

23 chromosome 1p/19q co-deletion (IDHmut-codel). Leveraging information theory and 
24 machine learning, we aim to quantify clonal stochasticity and track early molecular

25 predictors that drive cancer evolution.

\section{Longitudinal sequencing reveals mutational dynamics in glioma}

28 We assembled 511 matched initial and recurrent diffuse glioma pairs, among which 95 out

29 of 219 LGG cases progressed to GBM at recurrence. Out of these samples, 276 were

30 profiled by whole-genome sequencing (WGS), 989 by whole exome sequencing (WES),

31394 by RNA sequencing, and 201 by clinical panel sequencing (Extended Data Fig. 1a-

32 b, Extended Data Table 1-2). Using calibrated computational pipelines, we portrayed the

33 dynamic landscape of somatic mutations in key driver genes (Fig. 1a, Extended Data Fig.

34 1c-d). Consistent with previous reports $3,8,9,16$, we found that $17.0 \%$ (62 out of 365 ) of TMZ-

35 treated cases in our cohort gained hypermutation at recurrence. Excluding hypermutated

36 samples, elevated mutational burden at recurrence was observed in each of the three

37 molecular subtypes of glioma with $P$ values of $8.0 \times 10^{-6}, 2.9 \times 10^{-6}$ and $2.0 \times 10^{-3}$ for IDHwt,

38 IDHmut-noncodel, and IDHmut-codel (paired $t$-test), respectively (Extended Data Fig.

39 1e). Ternary plot showed that unlike IDHwt gliomas, IDH-mutant tumors had a remarkable

40 number of key alterations enriched in recurrent-specific area (Extended Data Fig. 2a-c).

41 In particular, 82 out of 128 IDHmut-noncodel and 13 out of 59 IDHmut-codel gliomas

42 developed recurrence-specific alterations in canonical GBM drivers such as CDKN2A

43 deletion and/or CDK4 amplification and deletions or mutations in PTEN (Extended Data

44 Fig. 2b-c). Interestingly, six recurrent IDHwt gliomas lost their initial alteration in

45 EGFRvIII and nine recurrent IDHwt gliomas lost the copy number amplification in MDM4.

46 Moreover, development of $\mathrm{HM}$ at recurrence was observed in all three subtypes, while one 
47 IDHwt and two IDHmut-noncodel cases developed MGMT translocation. These results

48 collectively implied that diffuse glioma recurred and developed chemoresistance in

49 response to current therapeutic intervention via divergent molecular mechanisms.

51 We next systematically compared genetic alterations of initial and recurrent tumors in all 52 patients and found that $70.2 \%$ of cases ( 269 out of 383 patients sequenced by WES/WGS)

53 lost $>5$ coding mutations (single nucleotide variants and small indels) while $81.9 \%$ (313

54 out of 383) cases gained $>5$ new mutations (Fig. 1b). For each molecular subtype, we

55 observed significantly larger number of recurrence-specific compared to initial-specific

56 mutations $\left(P=8.4 \times 10^{-7}, 6.3 \times 10^{-12}\right.$ and $1.5 \times 10^{-4}$ for IDHwt, IDHmut-noncodel, and

57 IDHmut-codel, respectively) (Fig. 1b). To quantify the changeability of each glioma

58 feature during evolution, we defined its longitudinal divergence by binarizing the change

59 of its mutational status over time and calculating the information entropy (Fig. 1c). As

60 expected, no longitudinal change was observed for mutational status of IDHI or co-

61 deletion of $1 \mathrm{p} / 19 \mathrm{q}$ and hence these features contained zero entropy, implying that these

62 alterations either achieved fixation due to their overwhelming survival advantage or

63 occurred extremely early during glioma evolution (Fig. 1d, Extended Data Fig. 2d-f). In

64 contrast, the pathologic grade, somatic hypermutation (HM), together with genomic

65 deletions in $C D K N 2 A, R B 1$ and PTEN contained the highest entropy, implying these

66 features changed frequently over time (Fig. 1d). We reason that the change of mutational

67 status of a glioma feature was not only determined by stochasticity, but also related to

68 potential predisposition factors observed early. Therefore, we calculated mutual

69 information between each feature's longitudinal divergence and data acquired from initial 
70 tumor to quantify predictability of the corresponding feature (Methods). Interestingly,

71 pathological grading, and HM are two features with the highest predictability, implying

72 these two events might be deployed earlier (Fig. 1d, Extended Data Fig. 2g-h).

\section{Genomic characteristics of initial glioma informs grade progression}

75 We then investigated the longitudinal change of pathologic grade in our cohort. Not

76 surprisingly, we found that patients who progressed to GBM showed significantly worse

77 survival compared to those who recurred as LGG, regardless of their molecular subtypes

78 (Fig. 2a). The above information theory analysis suggested that the risk of glioma grade

79 progression was related to early genetic and clinical factors. We therefore developed a

80 machine learning model to predict grade progression (LGG to GBM) from genomic and

81 clinical characteristics collected at diagnosis.

83 To develop the machine learning model, we compiled data of 116 LGG cases as the training

84 cohort (Fig. 2b, Extended Data Table 1). Particularly, the training cohort included 53

85 LGG-GBM longitudinal pairs together with 63 LGG-LGG pairs. Overall, three

86 classification models were trained using different sets of features: Model 1 used the initial

87 grade as the single predictor, Model 2 used the initial grade, age, gender, and treatment as

88 predictors, while Model 3 included the initial grade, age, gender, treatment, and genomic

89 alterations as predictors. The performance of the models was measured by the area under

90 the receiver operating characteristic curve (AUC). Based on five-fold cross validation we

91 found that the model using integrated features including patient clinical information,

92 applied treatment plus genomic alterations achieved the highest AUC $(0.87,95 \%$ CI 0.81 - 
93 0.94, DeLong test, Fig. 2c). The SHAP (Shapley additive explanations) score, a metric to

94 quantify the importance of each feature ${ }^{17,18}$, was then calculated and used to prioritize the

95 relevant features. On the top of the list is IDH status, where IDH mutation contributed

96 negatively to grade progression, and IDH wildtype are more prone to progress. Following

97 IDH mutations are $17 \mathrm{p}$ copy neutral loss of heterozygosity (CNLOH), $1 \mathrm{p} / 19 \mathrm{q}$ codel,

98 alkylator treatment and $C D K N 2 A$ deletion (Fig. 2d). Other relevant genetic factors include

99 amplification/gain of MET, chr7p and $M Y C$, deletions of TP53 and RB1, as well as TERT

100 promoter mutations. Notably, MYC, a master regulator of stemness and proliferation, has

101 been recently revealed to regulate glioma progression ${ }^{19}$.

102

103 To test the model performance, 50 independent LGG cases from GLASS were used as the

104 hold-out testing cohort. In this cohort, 21 LGGs progressed to GBM at recurrence and the

105 other 29 did not. Our model achieved an AUC of 0.79 (95\% confidence interval 0.67-0.92,

106 DeLong test; Fig. 2e). We then applied the model on 496 TCGA primary LGGs to assess

107 whether the predicted progression risk to GBM at recurrence might be associated with

108 patient survival outcome. As a result, remarkable worse overall survival (OS, $P=1.34 \times$

$10910^{-8}$, log-rank test) and progression-free survival (PFS, $P=1.15 \times 10^{-6}$, log-rank test)

110 outcomes were observed for cases predicted to progress to GBM, compared to those

111 predicted to recur as LGG (Fig. 2f). More importantly, when glioma patients were

112 evaluated in each of the three molecular groups, poorer PFS and OS were consistently

113 associated with predicted progression risk (Extended Data Fig. 3a-b). These findings

114 demonstrated that our machine learning model was able to integrate clinical information 
115 and molecular features in order to evaluate the risk of glioma progression and early predict 116 patient prognosis.

\section{Features of initial tumors predispose hypermutation at recurrence}

119 We adopted CELLO toolkit ${ }^{20}$ to infer HM from multi-platform sequencing data and

120 identified $68 \mathrm{HM}$ samples with average mutation load 157.7 mutations per $\mathrm{Mb}$ and average

121 HM score 1.39 (Extended Data Fig. 4a). In our dataset, we demonstrated that HM was

122 associated with glioma progression and impacts post-progression survival (Extended Data

123 Fig. 4b-c). Consistent with previous studies ${ }^{16,21,22}$, most (16 out of 18 samples with

124 available MGMT methylation status, 88.9\%) of HM samples were MGMT methylated, and

12559 of $65(90.8 \%) \mathrm{HM}$ cases gained loss-of-function mutations in the mismatch repair

126 (MMR) pathway genes such as MSH6 and MSH2 (Extended Data Fig. 4d. We then

127 compared the transcriptional profile of TMZ-treated HM versus NHM recurrent gliomas.

128 As expected, MGMT expression is significantly lower in HM samples compared to the

129 alkylator-treated non-hypermutated glioma, while genes such as cyclin dependent kinase 2

130 (CDK2) and polo-like kinase 1 (PLK1) had significantly higher expression in HM

131 (Extended Data Fig. 4e). The elevated PLK1 level in HM glioma is compatible with

132 results of a previous study that applied PLK1 inhibitors in MMR-deficient glioma cell

133 lines $^{23}$. Subsequent gene set enrichment analysis revealed MYC targets V2 as the most

134 significantly up-regulated gene set (normalized enrichment score $(\mathrm{NES})=3.32, P=5.2 \times$

$13510^{-4}$, Extended Data Fig. 4f) in hypermutated recurrent glioma. Other most upregulated

136 pathways included DNA repair, E2F targets, G2M checkpoint, and MYC targets V1,

137 implying HM samples had more active transcription and proliferation ${ }^{24}$. 
139 To identify the molecular features at diagnosis that drive the occurrence of HM at

140 recurrence, we compared the transcriptome of initial gliomas that recurred as hypermutated

141 (pre-HM, $\mathrm{n}=21$ ) versus the initial gliomas that recurred as non-HM tumors (pre-NHM, $\mathrm{n}=$

142 143). Mimicking the recurrent tumors, we found significant upregulation of MYC targets,

143 G2M checkpoint, E2F targets and mitotic spindle pathways in pre-HM samples (Figure

144 S5a-b). MYC pathway activation is known to be associated with cell proliferation. Indeed,

145 the commonly used cell proliferation marker, MKI67, is significantly up regulated genes

146 in pre-HM gliomas $\left(P=1.54 \times 10^{-3}\right.$, Wilcoxon's rank-sum test, Extended Data Fig. 5c).

147 Immunohistochemistry staining of 67 gliomas showed that gliomas with high Ki-67

148 staining have significantly higher probability to develop HM after TMZ treatment $(P=$

149 0.04, Fisher's exact test, Extended Data Fig. 5d). $M G M T$ was down-regulated in pre-HM

150 samples, which is consistent with the observation that MGMT-methylated gliomas, when

151 treated with TMZ, were prone to develop hypermutation $(P=0.021$, Fisher's exact test,

152 Extended Data Fig. 5e). Strikingly, comparing genomic alterations of pre-HM and pre-

153 non-HM gliomas we found that genomic gain of $M Y C$ was the most enriched feature in the

154 pre-HM tumors (fold change $=5.31, P=3.23 \times 10^{-5}$, Fisher's exact test), well in line with

155 the upregulation of MYC pathways (Fig. 3a-b). Specifically, about 46.9\% of MYC-gained

156 gliomas developed HM after TMZ treatment, while the proportion in gliomas without $M Y C$

157 gain was only $12.7 \%$ (Fig. 3c). Following MYC gain, other genomic alterations enriched

158 in initial tumors of hypermutators include $A T R X$ mutation and $R B 1$ deletion (Fig. 3a). Loss-

159 of-function mutations in $A T R X$ have been shown to impair DNA repair, leading to

160 genetically unstable tumors that rapidly accumulate oncogenic mutations ${ }^{25}$. RB1 encodes 
$161 \mathrm{Rb}$ protein and plays an essential role as a cell-cycle regulator acting at the G1/S cell cycle

162 checkpoint ${ }^{26}$. To make use of the comprehensive information for hypermutation prediction,

163 machine learning models were trained using genomic features of initial glioma plus

164 alkylator treatment information and achieved an Area Under Precision-Recall Curve

165 (AUPRC) of 0.88 . When the expression of $M G M T$ and MKI67 were included, the AUPRC

166 further improved to 0.95 (Fig. 3d). SHAP analysis showed that alkylator treatment was the

167 most predictive feature in the model, followed by MYC gain, ATRX, FUBPI and IDH

168 mutations, and EGFR amplification (Fig. 3e).

170 To interrogate the association between MYC and HM in glioma, we grew a MYC171 amplified glioma cell line U251 and a MYC-wild-type glioma cell line U87 in medium 172 containing escalating concentrations of TMZ (Extended Data Fig. 5f-g), until they 173 developed elevated TMZ resistance (TR; Extended Data Fig. 5h). During the whole 174 process, U251 maintained its high copy number and high expression of MYC gene, while 175 U87 had relatively low MYC expression (Extended Data Fig. 5i). Upon resistance, 176 U87TR showed elevated MGMT expression (Extended Data Fig. 5j). On the contrary, 177 U251TR maintained low level of $M G M T$ expression but gained thousands of mutations. 178 Over $90 \%$ of $\mathrm{U} 251 \mathrm{TR}$ mutations were $\mathrm{C}>\mathrm{T}(\mathrm{G}>\mathrm{A})$ single nucleotide variants, including an 179 MSH6 T1219I hotspot mutation with allele frequency 38\%, demonstrating the emergence 180 of the TMZ-induced hypermutation in vitro (Extended Data Fig. 5i-j). Considering U87 181 and U251 might not perfectly represent glioma patients, we further carried out the TMZ 182 inducing experiment in the newly derived gliomasphere (T2-4) sample from a patient with 183 high MYC copy number and expression (Fig. 3f). This sample developed dramatic TMZ 
184 resistance after the induction experiment (Fig. 3g). Interestingly, T2-4TR developed

185 hypermutation with a stop codon mutation $M L H 1 \mathrm{~W} 597 *(\mathrm{AF}=23 \%)$ and a splicing-donor 186 mutation (MLH1 c. $453+1 \mathrm{G}>\mathrm{A}, \mathrm{AF}=32 \%$, Fig. 3h), while maintaining a low level of 187 MGMT expression and a high level of MYC copy number and expression. Notably, the 188 splicing-donor mutation in $M L H 1$ caused an aberrant splicing which included an additional 189 nine basepairs in the spliced RNA (Fig. 3i). We next tracked the development of 190 hypermutator phenotype during T2-4 cell line passaging, and found the percent spliced in 191 (PSI) of the aberrant isoform emerged at passage six and increased over time (Fig. 3i).

192 Whole-exome sequencing of different passages demonstrated that hypermutation occurred 193 almost simultaneously with loss-of-function mutations in MLH1 at the sixth-seventh 194 passage of cell culture (Fig. 3i-j), confirming the underlying association between a burst 195 of somatic mutation and the MMR deficiency in glioma cells.

197 Hypermutation occurs frequently in actively transcribed genomic regions with MYC 198 binding

199 To explore the mechanism of how MYC activation promotes somatic hypermutation, we 200 first characterized the genomic distribution of hypermutation sites in relation to epigenetic 201 regulatory elements. The human genome was annotated using chromosomal binding peaks 202 from chromatin immunoprecipitation sequencing (ChIP-seq) data (Extended Data Fig. 203 Table 3, Methods), and then the mutational density of the TMZ-associated hypermutations 204 (HM) and non-TMZ-associated conventional somatic mutations (NHM) were calculated 205 and normalized for comparison. Consistent with previous observations that conventional 206 somatic mutations in the cancer genomes were not randomly distributed but enriched in 
207 transcriptionally inactive regions ${ }^{27-31}$, we found remarkably higher density of NHM 208 mutations around the H3K9me3 modification sites which are often associated with 209 heterochromatin (Fig. 4a, upper panel). In contrast, the density of hypermutations in these

210 regions were close to random. Conversely, while NHM mutations were significantly lower

211 than expected in the open chromatin regions marked by DNase hypersensitivity, HM had

212 remarkably higher mutation (Fig. 4a, lower panel). More importantly, the HM mutation

213 was significantly enriched in the periphery of $\mathrm{H} 3 \mathrm{~K} 27 \mathrm{ac}$ and $\mathrm{H} 3 \mathrm{~K} 4 \mathrm{me} 3$ sites that

214 respectively mark active enhancers and promoters, while NHM was of lower density (Fig.

$215 \mathbf{4 b}$, upper panel). In regions surrounding binding sites of RNA polymerase II (RPII), a key

216 enzyme in the transcriptional machinery, the density of HM mutations was almost two-fold

217 of NHM mutations (Fig. $\mathbf{4 b}$, lower left panel). It has been shown that in normal cells, the

218 actively transcribed genes recruit mismatch repair complex to protect the gene body from

219 mutation by $\mathrm{H} 3 \mathrm{~K} 36 \mathrm{me} 3^{32,33}$. We investigated the mutation densities around $\mathrm{H} 3 \mathrm{~K} 36 \mathrm{me} 3$

220 modification sites and noted that the mutation density of both HM and NHM mutations

221 were lower than random control, but the density of HM was much higher than NHM (Fig. $222 \mathbf{4 b}$, lower right panel). Altogether, these results demonstrated that in TMZ-associated 223 hypermutators, mutations were enriched to the proximity of active transcription sites.

225 To explore the role of MYC gain in hypermutagenesis, we compared MYC gene expression 226 between samples with and without MYC gain and found significantly higher MYC 227 expression in gliomas with MYC gain $\left(P=1.4 \times 10^{-7}\right.$, Wilcoxon rank-sum test; Extended 228 Data Fig. 6a). In tumor cells with elevated MYC expression, c-Myc forms a heterodimer 229 with MAX and binds to the promoters and enhancers of active genes, causing global 
230 transcription amplification ${ }^{34}$. To quantify the global transcriptional activity, we used

231 fourteen housekeeping genes as reference and counted the number of high-expression

232 genes (Methods). As expected, more high expression genes were observed in MYC-gain-

233 positive gliomas $\left(P=1.0 \times 10^{-3}\right.$, Wilcoxon rank-sum test, Fig. $\left.4 \mathbf{c}\right)$, indicating globally

234 amplified transcription in these samples. Comparison of the expression level of MYC direct

235 targets also showed consistent results $\left(P=1.3 \times 10^{-4}\right.$, Wilcoxon rank-sum test, Extended

236 Data Fig. 6b).

237

238 We next divided the open chromatin regions and active transcription sites based on whether

239 c-Myc also binds to the site, and then compared the density of HM mutations in these two

240 types of regions (Fig. 4d). The density of HM mutations was higher in open chromatin

241 with c-Myc binding compared to those without c-Myc binding. Similarly, when checking

242 H3K27ac modification sites with and without c-Myc binding, we found the mutation

243 density of HM to be much higher in sites with c-Myc binding than that in sites without c-

244 Myc binding (Fig. 4d). The same phenomenon was observed for H3K4me3 modification

245 sites, RPII binding sites and H3K36me3 modification sites with and without c-Myc binding 246 (Fig. 4d).

248 c-Myc binds MMR genes and increases probability of hypermutation

249 The eight MMR genes, namely PMS1, PMS2, MSH2, MSH3, MSH5, MSH6, MLH1 and $250 M L H 3$, all had H3K4me3 modification, c-Myc/MAX and RPII binding, especially in the 251 periphery of the promoter regions (Fig. 4e). In U87MG cell line, we experimentally 252 induced MYC overexpression and confirmed that MYC overexpression upregulated the 
253 expression of MSH6 and MSH2, the two most frequently altered MMR genes in HM (Fig.

254 4f, Extended Data Fig. 6c). Chi-square test with Yates' correction revealed significant 255 enrichment of MMR genes in c-Myc binding targets $(P=0.0002$, Fig. 4g), a group which

256 was highly enriched for temozolomide-driven mutations. Therefore, we propose that MYC

257 over activation increased the density of TMZ-induced mutations in the genomic regions of

258 MMR genes. With an elevated risk of developing loss-of-function mutations in MMR

259 genes, MYC-amplified gliomas hereby tend to develop HM in response to TMZ treatment.

260 Mechanistically, the double strand DNA is opened during transcription, and MAX and c-

261 Myc binding at the active promoters prolonged the time window of opening status. Under

262 TMZ treatment, the drug molecules attack the single stranded DNA, causing more damages

263 in the transcription active regions. When such damages occurred in MMR genes and failed

264 for repair, mutations will accumulate in other open sites and finally lead to hypermutation

265 (Fig. 4h). 
Discussion

267 The high rate of recurrence represents the major obstacle in improving survival of adult 268 diffuse glioma patients. Here, we have assembled and analyzed a mostly original cohort of 269511 cases with longitudinal matched samples of gliomas. This work highlighted the

270 longitudinal trajectory of evolution for each of the three main subtypes of gliomas (IDH 271 mutant codel, IDH mutant non-codel, IDH wild type). Through the development of novel

272 computational approaches, we found that grade progression and gain of hypermutation are

273 largely predictable from the molecular features of the initial glioma. Following training on 274 clinical and genomic features, our machine learning model robustly distinguished LGGs 275 that tend to progress to GBM from those that recur as LGG. Among the features with 276 highest importance, $C D K N 2 A$ deletion, a key cell-cycle related alteration has been 277 correlated with aggressive disease and tumor enhancing. ${ }^{8,35}$ Another important feature that 278 emerged from the model was TP53 copy-neutral loss of heterozygosity. Despite the 279 importance and prevalence of TP53 inactivation in glioma, little is known about how TP53 280 loss of function alterations contribute to glioma malignancy. Other important contributing 281 factors in the model include alkylator treatment and MYC gain, both of which are 282 associated with hypermutation. The relevance of these alterations as drivers of glioma 283 aggressiveness is underscored by the significant association between hypermutation and 284 grade progression.

285

286 The most important finding that emerged from our work is the discovery of the gain-of287 function genomic alterations of MYC as drivers of the HM state at recurrence. Several 288 reports including our own suggested that therapy-associated hypermutations in recurrent 
289 glioma are driven by MMR deficiency ${ }^{9,13,16}$. In our cohort, MMR deficiency was found in $29086 \%$ of hypermutated gliomas at recurrence. Indeed, whereas MMR deficiency is the direct 291 causal factor leading to hypermutation, the absence of mutations in MMR genes in 292 untreated tumors suggested that other, upstream alterations might be necessary to set in 293 motion the machinery leading to the hypermutated state. The key early event we uncovered,

294 was gain of MYC, an event significantly detected in untreated glioma primed to recur as 295 hypermutated tumors. The role of MYC is especially significant in the context of the IDH 296 mutant non-codel subtype, which is also the glioma group with the highest likelihood to 297 undergo transformation to the hypermutated state at recurrence. Through the integration of 298 exome and transcriptomic analyses, we found that gain of MYC results in marked 299 activation in downstream pathways associated with proliferation and deregulated cell cycle 300 progression, which are the primary biological functions of MYC in mammalian cells. The 301 role of MYC as unexpected driver of the hypermutation state has been experimentally 302 validated in human glioma cells. Furthermore, by integrating genomic and epigenetic data, 303 we confirmed our previous hypothesis that mutagenic mechanisms related to TMZ 304 treatment and subsequent MMR alteration act more efficiently in highly expressed regions 305 of open chromatin ${ }^{16}$, and further showed that c-Myc binding augmented the chance of 306 MMR mutations. Interestingly, highly expressed regions are protected by transcription307 coupled repair (TCR) $)^{36}$, and hypermutated tumors exhibited an enrichment of somatic 308 mutations in TCR genes in the hypermutated glioma samples. However, whether the TCR 309 pathway is functional in these samples will have to be established by future studies. 
311 Collectively, this study painted the evolution routes of three molecular types of glioma,

312 trained machine learning models to predict grade progression based on clinical and

313 genomic features of initial glioma and demonstrated MYC as a potential predictor of

314 hypermutation after treatment. In addition, we have developed an interactive, publicly

315 available web resource (Extended Data Fig. 7; https://wanglab.shinyapps.io/cello/) to

316 explore the longitudinal glioma dataset and make predictions of developing treatment-

317 induced hypermutation and grade progression.

318 
320 This work was supported by NSFC Excellent Young Scientists Fund (Hong Kong and

321 Macau) (No. 31922088), RGC grants (N_HKUST606/17, 26102719), ITC grant

322 (ITCPD/17-9), National Natural Science Foundation of China (NSFC)/Research Grants

323 Council (RGC) Joint Research Scheme (No. 81761168038), National Natural Science

324 Foundation of China (No. 81903078, No. 81972816), National Key Research and

325 Development Project of China (No. 2019YFE0109400), Hong Kong Epigenomics Project

326 (LKCCFL18SC01-E), and a grant from the Korea Health Technology R\&D through the

327 Korea Health Industry Development Institute funded by the Ministry of Health \& Welfare,

328 Republic of Korea (HI14C3418). The authors would like to thank all contributors to the

329 Chinese Glioma Genome Atlas. In addition, the authors acknowledge data generators of

330 the published datasets, especially TCGA research network, the GLASS Consortium,

331 Gregory M. Kiez and Mehmet Kutman Foundation, and the Yale University Department

332 of Neurosurgery for providing access to the raw and/or processed sequencing data.

\section{Author Contributions}

335 J.W., T.J., D.H.N., and A.I. conceptualized and supervised the project. Q.M. performed the

336 computational and statistical analyses; R.C., H.L. and Y.C. carried out the cell line and

337 PDC experiments; Y.Y. and Q.M. developed the CELLO webserver; Z.Z., Z.B., and T.J.

338 contributed to sample preparation, genomic sequencing, and clinical data collection of the

339 CGGA cohort; M.H.L., D.S. and B.J. contributed to some of the bioinformatics analyses;

340 J.K.S., H.J.C., and D.H.N. contributed to sample preparation, genomic sequencing and

341 clinical data collection of the SMC cohort; A.C., W.S.P., H.K.N., and D.M.C. collected the 
342 CUHK samples; K.C., D.L, S.T, and A.W. prepared libraries for samples of the CUHK

343 cohort; J.W. and Q.M. wrote the manuscript, which was then revised and proof-read by all

344 authors.

345

346 Competing Interests

347 The authors declare no potential conflict of interests. 


\section{References}

349 1. Greaves, M. \& Maley, C. C. Clonal evolution in cancer. Nature 481, 306-313 (2012).

2. McGranahan, N. \& Swanton, C. Clonal Heterogeneity and Tumor Evolution: Past, Present, and the Future. Cell (2017). doi:10.1016/j.cell.2017.01.018

3. Hu, H. et al. Mutational Landscape of Secondary Glioblastoma Guides METTargeted Trial in Brain Tumor. Cell 175, 1665-1678.e18 (2018).

4. Lee, J.-K. et al. Spatiotemporal genomic architecture informs precision oncology in glioblastoma. Nat. Genet. 49, 594-599 (2017).

5. Kim, J. et al. Spatiotemporal Evolution of the Primary Glioblastoma Genome. Cancer Cell 28, 318-328 (2015).

6. Mazor, T. et al. DNA Methylation and Somatic Mutations Converge on the Cell Cycle and Define Similar Evolutionary Histories in Brain Tumors. Cancer Cell 28, 307-317 (2015).

7. Suzuki, H. et al. Mutational landscape and clonal architecture in grade II and III gliomas. Nat. Genet. 47, 458-468 (2015).

8. Barthel, F. P. et al. Longitudinal molecular trajectories of diffuse glioma in adults. Nature 576, 112-120 (2019).

9. Johnson, B. E. et al. Mutational Analysis Reveals the Origin and Therapy-Driven Evolution of Recurrent Glioma. Science (80-. ). 343, 189-193 (2014).

10. Bai, H. et al. Integrated genomic characterization of IDH1-mutant glioma malignant progression. Nat. Genet. 48, 59-66 (2016).

11. Wang, Q. et al. Tumor Evolution of Glioma-Intrinsic Gene Expression Subtypes Associates with Immunological Changes in the Microenvironment. Cancer Cell 32, 42-56.e6 (2017).

12. Schwartzbaum, J. A., Fisher, J. L., Aldape, K. D. \& Wrensch, M. Epidemiology and molecular pathology of glioma. Nature Clinical Practice Neurology 2, 494503 (2006).

13. Touat, M. et al. Mechanisms and therapeutic implications of hypermutation in gliomas. Nature 580, 517-523 (2020).

14. Cipponi, A. et al. MTOR signaling orchestrates stress-induced mutagenesis, facilitating adaptive evolution in cancer. Science 368, 1127-1131 (2020).

15. Oldrini, B. et al. MGMT genomic rearrangements contribute to chemotherapy resistance in gliomas. Nat. Commun. 11, 1-10 (2020).

16. Wang, J. et al. Clonal evolution of glioblastoma under therapy. Nat. Genet. 48, 768-776 (2016).

17. Lundberg, S. M. et al. From local explanations to global understanding with explainable AI for trees. Nat. Mach. Intell. 2, 56-67 (2020).

18. Lundberg, S. M. \& Lee, S.-I. A Unified Approach to Interpreting Model Predictions. in Advances in Neural Information Processing Systems (eds. Guyon, I. et al.) 30, 4765-4774 (Curran Associates, Inc., 2017).

19. Bai, H. et al. Integrated genomic characterization of IDH1-mutant glioma malignant progression. Nat. Genet. 48, 59-66 (2016).

20. Jiang, B., Song, D., Mu, Q. \& Wang, J. CELLO: a longitudinal data analysis toolbox untangling cancer evolution. Quant. Biol. 8, 256-266 (2020). 
21. van Thuijl, H. F. et al. Evolution of DNA repair defects during malignant progression of low-grade gliomas after temozolomide treatment. Acta Neuropathol. 129, 597-607 (2015).

22. Mathur, R. et al. MGMT promoter methylation level in newly diagnosed lowgrade glioma is a predictor of hypermutation at recurrence. Neuro. Oncol. (2020). doi:10.1093/neuonc/noaa059

23. Higuchi, F. et al. PLK1 inhibition targets MYC-activated malignant glioma cells irrespective of mismatch repair deficiency-mediated acquired resistance to temozolomide. Mol. Cancer Ther. 17, 2551-2563 (2018).

24. Liberzon, A. et al. The Molecular Signatures Database Hallmark Gene Set Collection. Cell Syst. 1, 417-425 (2015).

25. Koschmann, C. et al. ATRX loss promotes tumor growth and impairs nonhomologous end joining DNA repair in glioma. Sci. Transl. Med. 8, 328ra28328ra28 (2016).

26. Bertoli, C., Skotheim, J. M. \& De Bruin, R. A. M. Control of cell cycle transcription during G1 and S phases. Nature Reviews Molecular Cell Biology 14, 518-528 (2013).

27. Lawrence, M. S. et al. Mutational heterogeneity in cancer and the search for new cancer-associated genes. Nature 499, 214-218 (2013).

28. Polak, P. et al. Cell-of-origin chromatin organization shapes the mutational landscape of cancer. Nature 518, 360-364 (2015).

29. Gonzalez-Perez, A., Sabarinathan, R. \& Lopez-Bigas, N. Local Determinants of the Mutational Landscape of the Human Genome. Cell 177, 101-114 (2019).

30. Makova, K. D. \& Hardison, R. C. The effects of chromatin organization on variation in mutation rates in the genome. Nature Reviews Genetics 16, 213-223 (2015).

31. Akdemir, K. C. et al. Somatic mutation distributions in cancer genomes vary with three-dimensional chromatin structure. Nat. Genet. 52, 1178-1188 (2020).

32. Huang, Y., Gu, L. \& Li, G. M. H3K36me3-mediated mismatch repair preferentially protects actively transcribed genes from mutation. J. Biol. Chem. 293, 7811-7823 (2018).

33. Li, F. et al. The histone mark $\mathrm{H} 3 \mathrm{~K} 36 \mathrm{me} 3$ regulates human DNA mismatch repair through its interaction with MutSa. Cell 153, 590-600 (2013).

34. Lin, C. Y. et al. Transcriptional amplification in tumor cells with elevated c-Myc. Cell 151, 56-67 (2012).

35. Jonsson, P. et al. Genomic correlates of disease progression and treatment response in prospectively characterized gliomas. Clin. Cancer Res. 25, 5537-5547 (2019).

36. Hanawalt, P. C. \& Spivak, G. Transcription-coupled DNA repair: Two decades of progress and surprises. Nature Reviews Molecular Cell Biology 9, 958-970 (2008).

37. Kim, H. et al. Whole-genome and multisector exome sequencing of primary and post-treatment glioblastoma reveals patterns of tumor evolution. Genome Res. 25, 316-327 (2015).

38. Ceccarelli, M. et al. Molecular Profiling Reveals Biologically Discrete Subsets and Pathways of Progression in Diffuse Glioma. Cell 164, 550-563 (2016).

39. Zhao, J. et al. Immune and genomic correlates of response to anti-PD-1 
40. Hu, H. et al. Mutational Landscape of Secondary Glioblastoma Guides METTargeted Trial in Brain Tumor. Cell 175, 1665-1678.e18 (2018).

41. Lee, J.-K. et al. Pharmacogenomic landscape of patient-derived tumor cells informs precision oncology therapy. Nat. Genet. 50, 1399-1411 (2018).

42. Trifonov, V., Pasqualucci, L., Tiacci, E., Falini, B. \& Rabadan, R. SAVI: A statistical algorithm for variant frequency identification. BMC Syst. Biol. 7, S2 (2013).

43. Cingolani, P. et al. A program for annotating and predicting the effects of single nucleotide polymorphisms, SnpEff. Fly (Austin). 6, 80-92 (2012).

44. Sherry, S. T. et al. dbSNP: the NCBI database of genetic variation. Nucleic Acids Res. 29, 308-11 (2001).

45. Tate, J. G. et al. COSMIC: the Catalogue Of Somatic Mutations In Cancer. Nucleic Acids Res. 47, D941-D947 (2019).

46. Talevich, E., Shain, A. H., Botton, T. \& Bastian, B. C. CNVkit: Genome-Wide Copy Number Detection and Visualization from Targeted DNA Sequencing. PLOS Comput. Biol. 12, e1004873 (2016).

47. Li, H. et al. The Sequence Alignment/Map format and SAMtools. Bioinformatics 25, 2078-2079 (2009).

48. Haas, B. J. et al. Accuracy assessment of fusion transcript detection via readmapping and de novo fusion transcript assembly-based methods. Genome Biol. 20, 213 (2019).

49. Wang, J. et al. Tumor evolutionary directed graphs and the history of chronic lymphocytic leukemia. Elife 3, (2014).

50. Chen, T. \& Guestrin, C. XGBoost: A Scalable Tree Boosting System. in Proc. 22nd ACM SIGKDD International Conference on Knowledge Discovery and Data Mining 785-794 (2016). doi:10.1145/2939672.2939785 
a

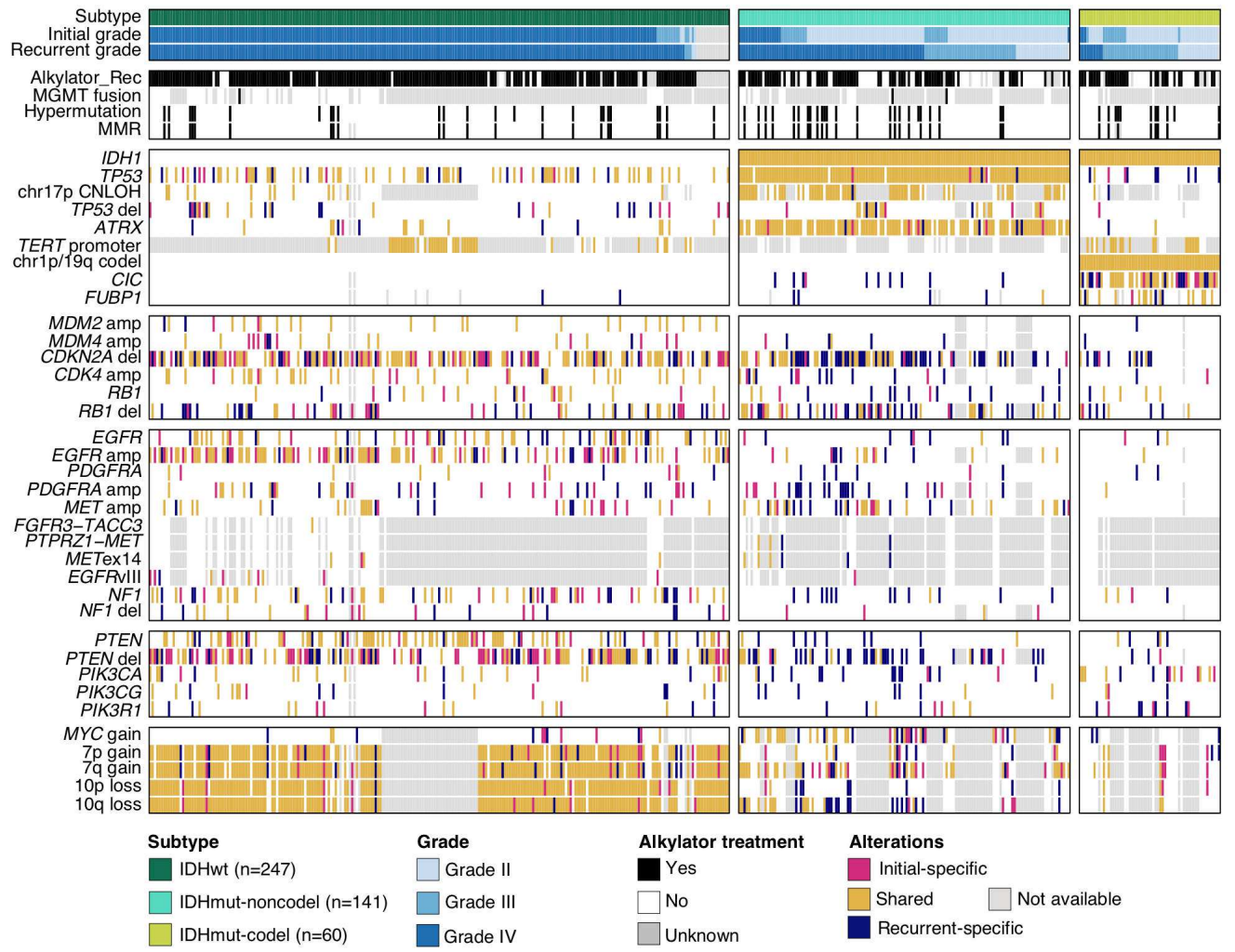

b

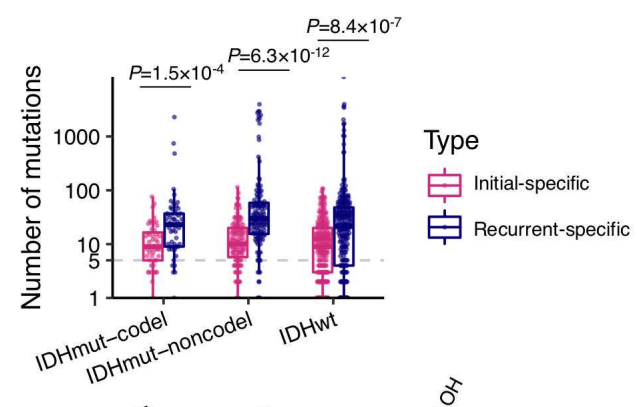

d

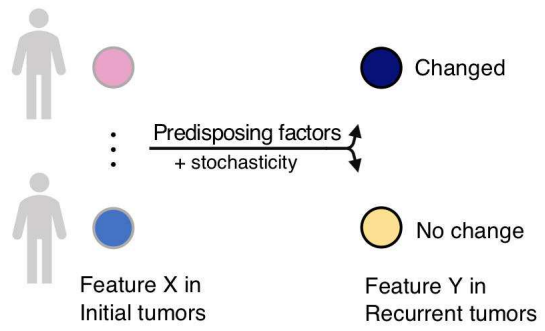

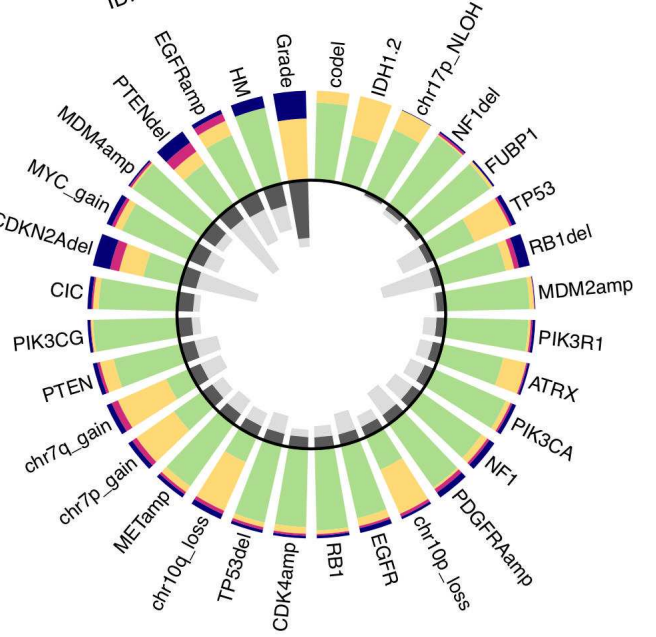
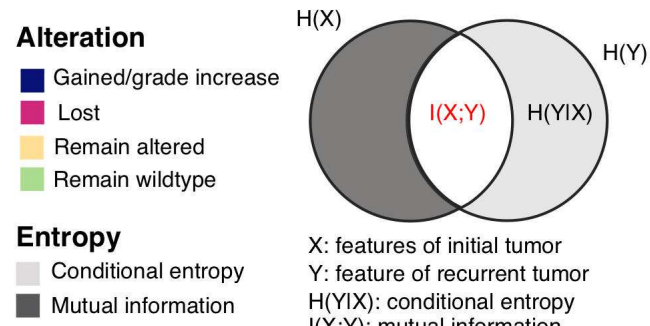

$X$ : features of initial tumor

$Y$ : feature of recurrent tumor

$\mathrm{H}(\mathrm{Y} \mid \mathrm{X})$ : conditional entropy $\mathrm{I}(\mathrm{X} ; \mathrm{Y})$ : mutual information 
471 Figure 1. Identification of predictable events from pan-glioma evolutionary landscape.

472 (a) Longitudinal molecular landscape of glioma patients with paired DNA sequencing data.

473 Each column represents a patient which were stratified by the molecular subtype, and each

474 row represents a clinical feature or molecular variant. The color of each variant indicates

475 whether it is specific to initial, recurrence or shared. MMR: mismatch repair; CNLOH:

476 copy-neutral loss of heterozygosity. (b) Comparison of the number of initial- and recurrent-

477 specific somatic mutations during evolution in each glioma subtype. The horizontal dashed

478 line represents five mutations. The $P$ values were calculated by Wilcoxon's rank-sum test.

479 (c) Proposed model explaining the changes of the clinical and molecular features over time.

480 Predisposing factors in the initial tumor, together with stochasticity, determine whether the

481 status of the feature will change at recurrence. (d) Alteration status, entropy and mutual

482 information of clinical and genomic features. Stacked bars outside the circle summarizes

483 the frequency of a clinical feature or molecular variant to be lost, gain, remaining wildtype

484 or remaining altered. Inside the circle shows the overlapped bar plots of mutual information

485 (black) and conditional entropy (grey), where high proportion of overlap indicates better

486 predictability. The features are ranked by mutual information. 
a

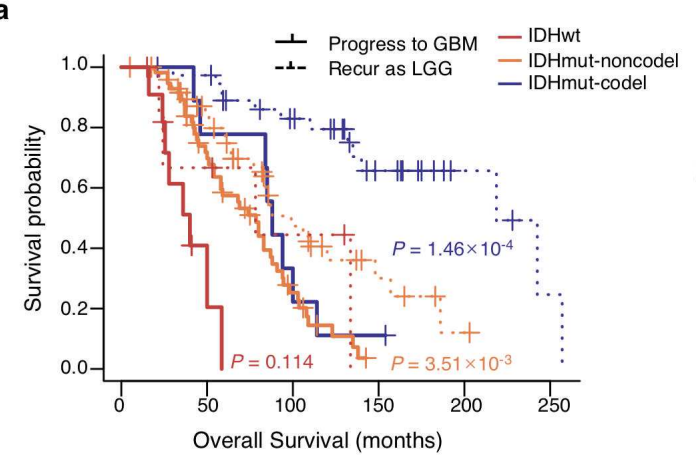

b

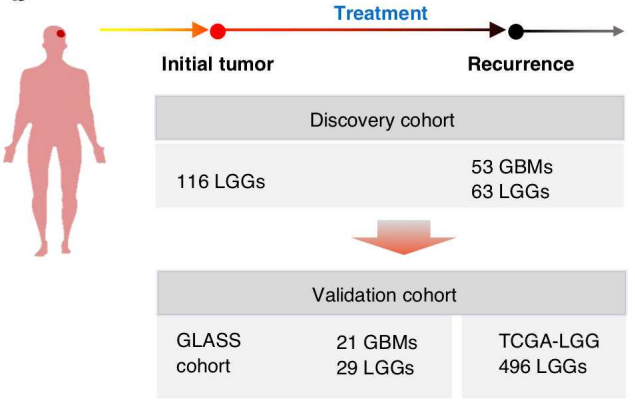

C

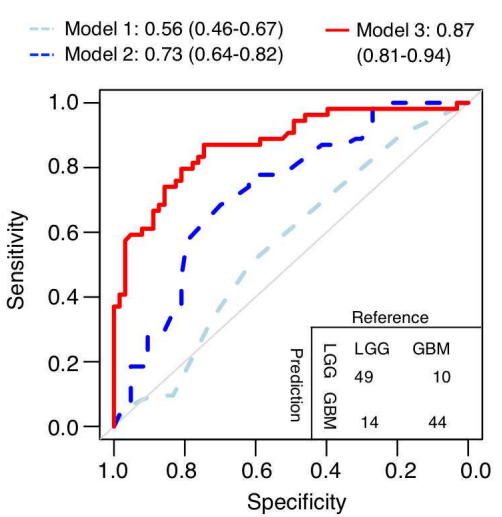

d

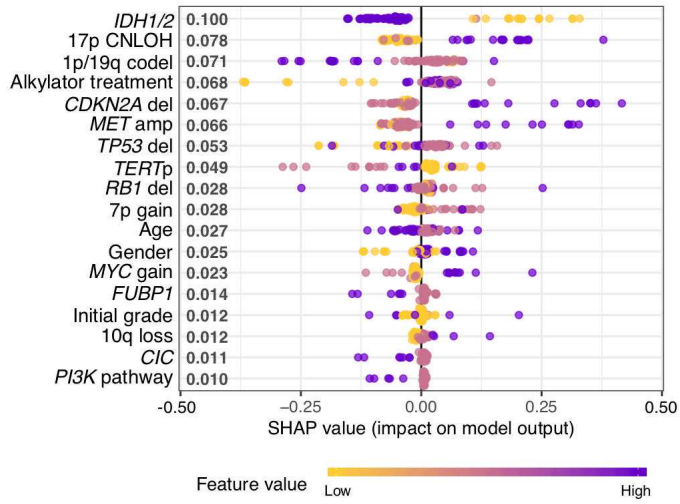

e

-- Model 1: 0.66 (0.54-0.78) - Model 3: 0.79

--. Model 2: $0.67(0.51-0.82) \quad(0.67-0.92)$

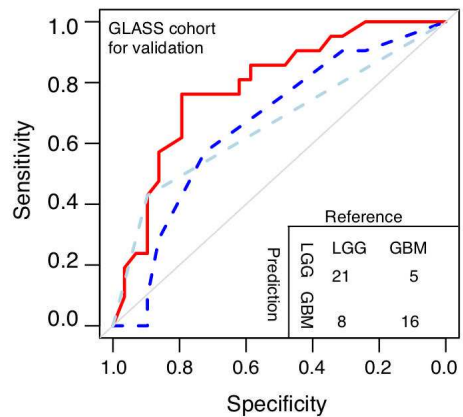

f

1 Predicted to progress $\perp$ Predicted to recur to $\mathrm{GBM}(\mathrm{n}=192)$

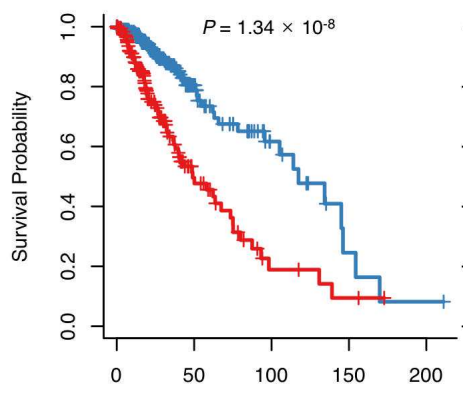

Overall survival (months)

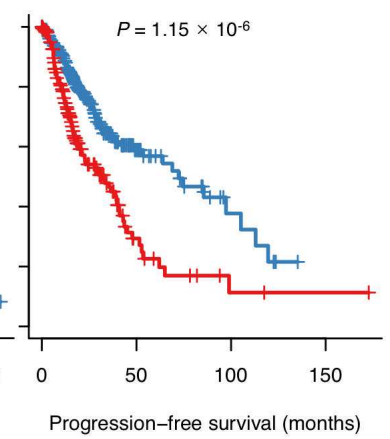

Figure 2. Genomic characteristics of initial glioma informs grade progression after

490 (a) Overall survival of low-grade gliomas that progressed to glioblastoma (GBM) and those

491 that recurred as low-grade glioma (LGG). The colors represent glioma subtypes, while the

492 line type (solid or dashed) show whether the patient progressed to GBM at recurrence. The 
$493 \quad P$ values were calculated by log-rank test. (b) Design of the machine learning tasks. The 494 discovery cohort, including 117 LGGs, was used to train machine learning models to 495 predict whether the initial LGG patients would progress to GBM at recurrence. The models 496 were then applied to two independent cohorts for validation. (c) Receiving operation 497 characteristic (ROC) curves showing the cross-validation performance of the models 498 trained using different combinations of features. Model 1: the initial grade as the single 499 predictor; Model 2: the initial grade, age, gender, and treatment as predictors; Model 3: the 500 initial grade, age, gender, treatment, and genomic alterations as predictors. (d) Shapley 501 additive explanation (SHAP) scores of features in the prediction model of grade 502 progression. The color of the points represents value of each feature. (e) ROCs showing 503 the three models' prediction performance in the GLASS cohort. A confusion matrix is 504 shown at the right bottom. (f) Overall survival (left panel) and progression-free survival 505 (right panel) of LGGs from TCGA-LGG cohort, stratified by their predicted risk of 506 progressing to GBM. Patients that were in this longitudinal cohort were excluded. The $P$ 507 values were calculated by log-rank test. 
a
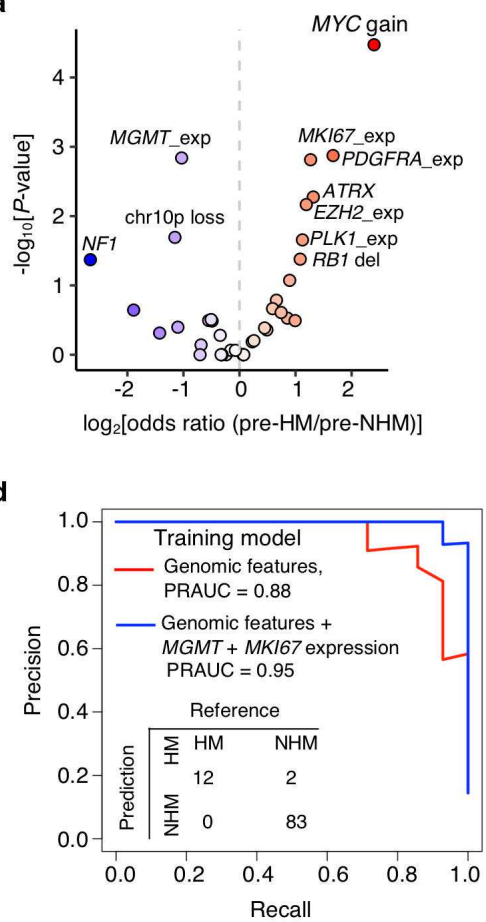

f
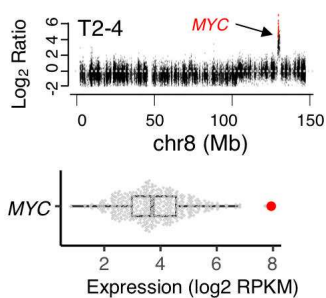

i

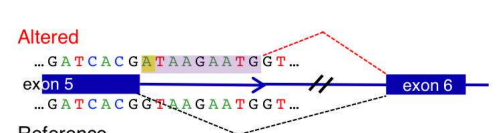

Reference

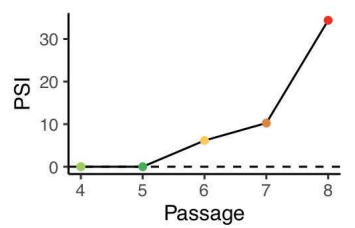

b
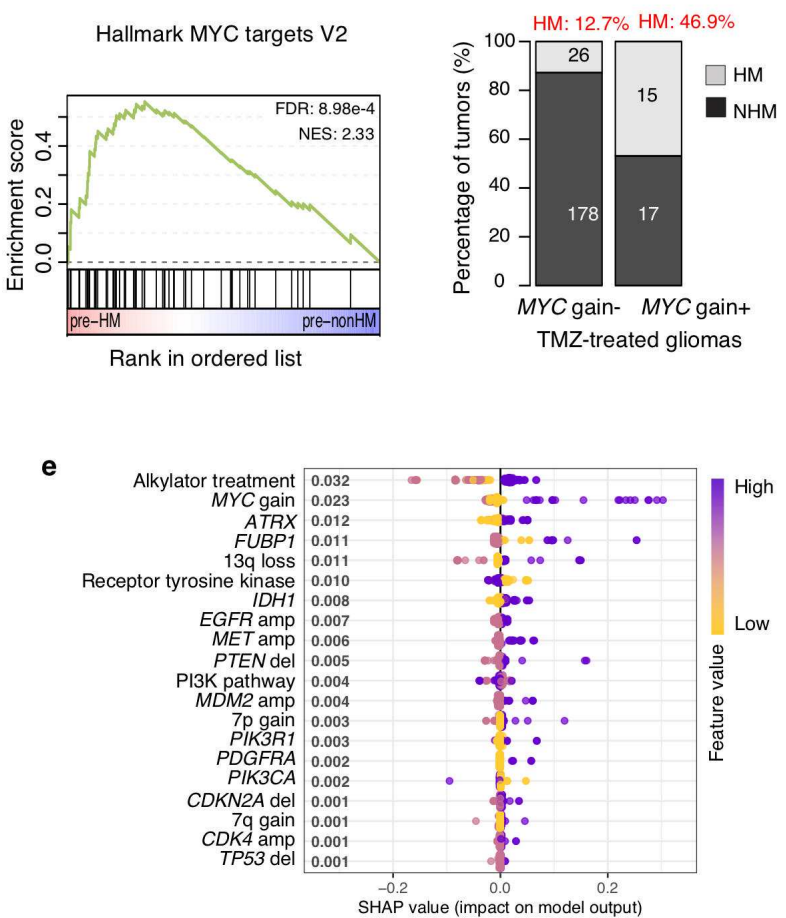

h

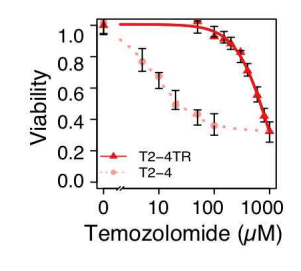

C

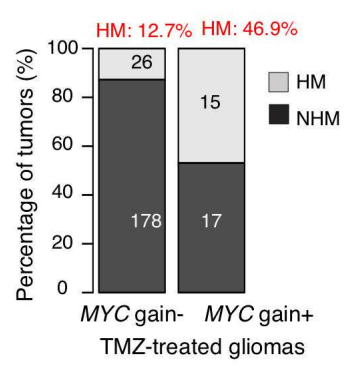

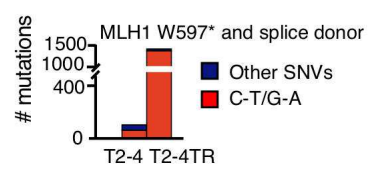

j

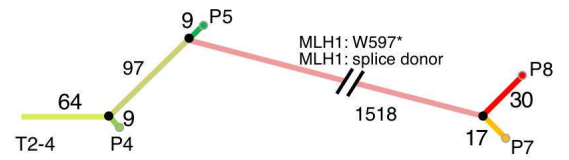

509 Figure 3. Features of initial tumors predispose TMZ-associated hypermutation at

510 recurrence.

511 (a) Comparison of genomic and transcriptomic features of the TMZ-treated initial gliomas

512 preceding hypermutators (pre-HM) and initial tumors preceding non-hypermutators (pre-

513 NHM). (b) Gene set enrichment analysis. Gene expression profile of pre-HM gliomas were 
514 compared to pre-NHM gliomas. FDR: false-discovery rate; NES: normalized enrichment 515 score. (c) The frequency of developing hypermutation in TMZ treated gliomas with or 516 without MYC copy number gain. (d) Precision-recall curve of HM prediction the model 517 trained using genomic features (red curve) and genomic features plus expression of $M G M T$ 518 and MKI67 (blue curve). (e) SHAP value of each feature in the model trained with genomic

519 features plus expression of $M G M T$ and MKI67 in (c). (f) The DNA copy number of MYC 520 in T2-4 (upper panel), and the expression level (lower panel) of T2-4 (the red dot) and other 521 RNA sequenced gliomas in this study (grey dots). (g) Response curves of T2-4 and the 522 induced T2-4TR cell line to TMZ. (h) Number of somatic mutations in T2-4 and T2-4 TR.

523 Dark red represents $\mathrm{C}$ to $\mathrm{T}$ or $\mathrm{G}$ to A mutations, while dark blue represents other somatic 524 mutations. (i) Aberrant splicing related to the MLH1 splicing donor mutation in T2-4. The 525 upper panel shows the aberrant splicing which included nine additional base pairs in the 526 spliced mRNA. The lower panel shows the percentage of spliced-in (PSI) reads of the 527 aberrant isoform in different passages of T2-4. (j) Mutations in T2-4 at different time points 528 during the TMZ-inducing experiment. The upper panel shows the phylogenetic tree 529 constructed from the mutations in passages $4,5,7$ and 8 as compared to the untreated T25304 PDC, and the lower panel shows a heatmap of the mutations. 
a

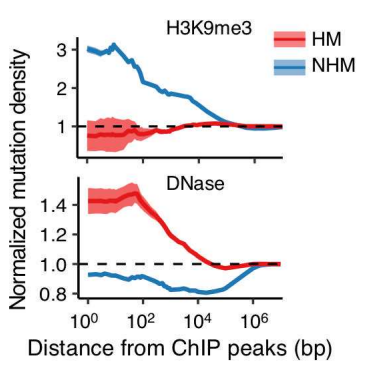

b

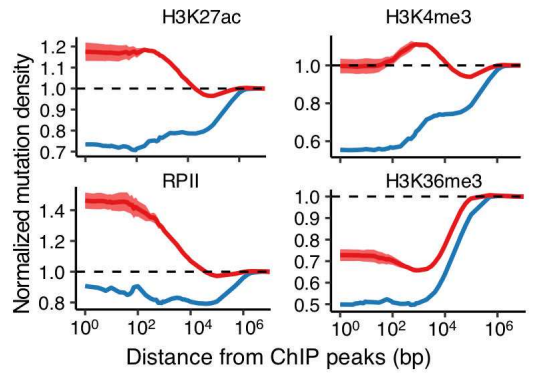

C

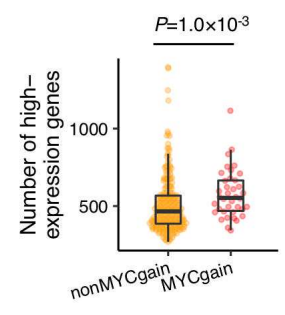

d

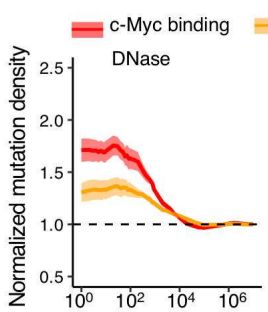

non-c-Myc binding
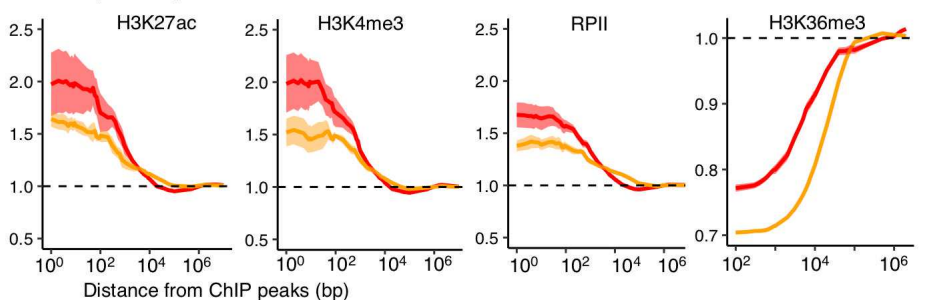

e
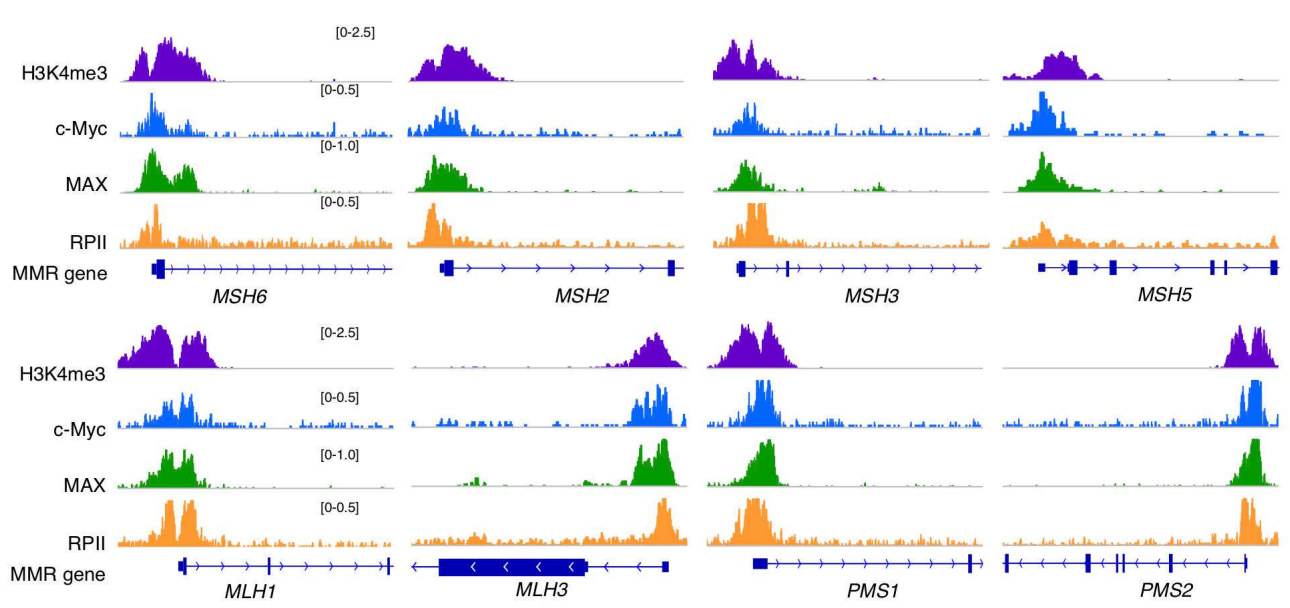

f

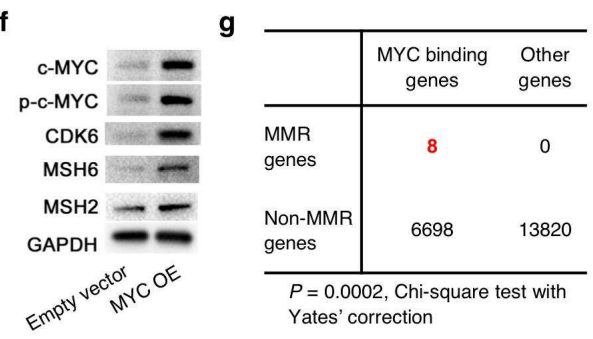

\section{h}

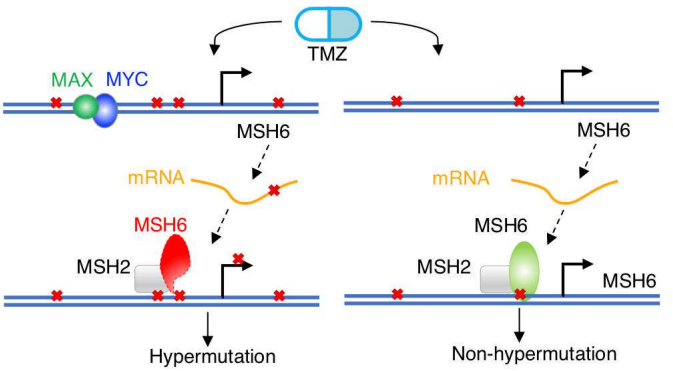

532 Figure 4. TMZ-induced mutations are enriched in open active regions, while c-Myc

\section{3 binding further increases mutation density}

534 (a) Normalized mutation density of TMZ-associated hypermutations (HM) and non-TMZ

535 associated recurrence-specific somatic mutations (NHM) around H3K9me3 modification

536 sites (upper panel) and DNase hypersensitive sites (lower panel). The dashed horizontal 
537 line represents the random level. (b) Normalized mutation density of HM and NHM around

538 H3K27ac modification sites (upper left), H3K4me3 modification sites (upper right), RNA

539 polymerase II (RPII) binding sites (lower left) and H3K36me3 modification sites (lower

540 right). (c) Number of high-expression genes in gliomas with and without MYC gain. The

$541 \quad P$ value was calculated by Wilcoxon's rank-sum test. (d) Normalized mutation density of

542 HM around DNase hypersensitive sites, H3K27ac modification sites, H3K4me3

543 modification sites, RPII binding sites and H3K36me3 modification sites with (red color)

544 and without (orange color) c-Myc binding. (e) Chromatin immunoprecipitation (ChIP)

545 intensity of H3K4me3 modification, c-Myc, MAX and RPII binding at eight mismatch

546 repair (MMR) genes. (f) Contingent table of c-Myc binding and MMR genes. (g) Western

547 blot showing c-MYC, p-c-MYC, CDK6, MSH6, MSH2, and MLH1 in U87 cell lines with

548 empty vector or MYC overexpression. OE: overexpression. (h) Proposed working model

549 of how MYC activation increases chance of hypermutation. 


\section{Methods}

\section{Human Patients and Samples}

3 In this study we collected longitudinally paired glioma from at least two time points,

4 namely the initial tumor and the recurrent tumor. Where possible, blood samples were also

5 collected. The newly collected samples are from Capital Medical University Beijing

6 Tiantan Hospital (China), Samsung Medical Center (SMC, Korea) and CUHK Otto Wong

7 Brain Tumour Centre in Hong Kong.

8 Glioma patients from Beijing Tiantan Hospital were enrolled as part of the Chinese Glioma

9 Genome Atlas project (CGGA, http://cgga.org.cn). Ethical approval was obtained from the

10 institutional review board in Capital Medical University (IRB ID: KYSQ2019-200-01),

11 and informed consent for each patient was obtained before surgery. For each specimen, the

12 pathological diagnosis is confirmed independently by two neuropathologists based on the

132016 edition of WHO classification of central nervous system tumors. The specimen was

14 flash-frozen within 5 mins after resection and restored in liquid nitrogen until being used

15 for DNA/RNA extraction and other experiments.

17 The SMC cohort includes both published cases and newly enrolled samples. The study is 18 approved by the institutional review board of SMC (IRB file No. 2010-04-004 \& 2005-04-

19 001), and written consent is obtained from each patient. The glioma specimens were 20 immediately snap-frozen after surgical resection and stored in in liquid nitrogen for further

21 analysis. This study also included samples from six patients with recurrent glioma from

22 CUHK Otto Wong Brain Tumour Centre. Ethical approval was obtained from the Joint 
23 Chinese University of Hong Kong-New Territories East Cluster Clinical Research Ethics

24 Committee with Reference No. 2018.389.

26 In addition to these newly collected specimens, we also curated sequencing data of

27 longitudinally paired glioma from published cohorts. This include the Istituto Neurologico

28 C. Besta cohort (INCB, $\mathrm{n}=19)^{16}$, the MD Anderson Cancer Center cohort (MD Anderson,

$29 \mathrm{n}=10)^{37}$, the Kyoto University cohort $(\mathrm{KU}, \mathrm{n}=10)^{7}$, the University of California San

30 Francisco cohort (UCSF, $n=23)^{9}$, The Cancer Genome Atlas cohort (TCGA, $\left.n=27\right)^{38}$, the

31 Yale cohort $(n=41)^{19}$, the Columbia University cohort (Columbia, $\left.n=14\right)^{39}$, the Memorial

32 Sloan Kettering Cancer Center cohort (MSKCC, $\mathrm{n}=67)^{35}$. The original Glioma

33 Longitudinal AnalySiS (GLASS) $\operatorname{cohort}^{8}$ included some samples from the above collection,

34 so we included the rest 139 patients for which sequencing data of both initial and recurrent

35 tumors were available and designated as GLASS cohort.

\section{Sample processing and Illumina sequencing}

38 For the samples from Tiantan hospital, DNA and/or RNA from the tumor were extracted

39 using the same protocol as described previously ${ }^{15,40}$. Where available, DNA from matched

40 blood was also extracted. For patients PS115-PS132, the exome regions of both tumor and

41 blood DNA were captured using the Agilent SureSelect XT Human All ExonV5 kit, and

42 then sequenced using Illumina HiSeq 2500 platform. For patients PS132-PS157, whole

43 genome libraries were prepared and sequenced using Illumina HiSeq 2500 platform,

44 generating about $180 \mathrm{~Gb}$ and $90 \mathrm{~Gb} 150 \mathrm{bp}$ paired end reads for tumor and normal DNA,

45 respectively. For RNA, the extracted total RNA was firstly depleted for tRNA and rRNA, 
46 then reverse-transcribed to cDNA, and sequenced using Illumina HiSeq 2500 platform,

47 generating around $6 \mathrm{~Gb} 150 \mathrm{bp}$ paired end reads for each sample.

49 Samples from SMC were processed and sequenced as previously described ${ }^{41}$. For samples

50 from CUHK, both total DNA and RNA were isolated from tumor samples while only total

51 DNA was purified from blood samples. Frozen tumors were homogenized in Buffer RLT

52 (Qiagen) with 40mM DTT and subsequently filtered by QIAshredder (Qiagen). Total

53 DNA/RNA from tissue lysate and from blood were then isolated using AllPrep DNA/RNA

54 Mini kit (Qiagen) according to manufacturer's protocol. The quantity and purity of the

55 nucleic acids were determined using Qubit 3 Fluorometer with either dsDNA HS Assay

56 Kit or RNA HS Assay Kit. For DNA samples with sub-optimal purity determined by

57 A260/280 ratio, they were further cleaned up using DNA Clean \& Concentrator-5 kit

58 (ZYMO Research). RNA integrity was assessed by RNA Quality Number (RQN) using

59 Fragment Analyzer (Advanced Analytical) with HS RNA Kit (15NT). DNA samples with

60 a A260/280 of $\sim 1.8$, and RNA samples with a A260/280 of $\sim 2.0$ and RQN $>5$ were

61 subjected to sequencing. Exome DNA were subsequently captured using Agilent

62 SureSelect XT Human All ExonV5 kit. DNA and RNA were sequenced using the same

63 protocol as samples from Tiantan Hospital, but generated 24Gb reads for tumor DNA, 12

$64 \mathrm{~Gb}$ reads for blood DNA, and $12 \mathrm{~Gb}$ reads for RNA.

65

66 For the published samples, a detailed list of the sequencing types and protocols were 67 summarized in Table S1.

68 


\section{Sequencing data preprocessing and mapping}

70 The quality of the sequencing reads was first checked by running FastQC v0.11.5. The low-

71 quality reads (containing at least one ambiguous base, or average quality $<20$ ) were then

72 removed using fastp v0.20.1. For DNA sequencing, the clean reads were mapped to the

73 human reference genome hg19 using Burrows Wheeler Aligner (BWA, v0.7.15-r1140)

74 mem algorithm, then sorted by coordinates using samtools v1.2. Subsequently, duplicate

75 reads were marked by running Picard MarkDuplicates 2.9.2 tool

76 (https://broadinstitute.github.io/picard/), generating the final bam file for mutation and

77 copy number identification. For RNA-seq, the reads were mapped to hg19 using STAR

78 2.6.1d. The gene annotation is ENSEMBL GRCh37.75.

79 For published samples, sequencing reads were either downloaded from NCBI SRA or

80 extracted from the published bam files that we downloaded from EGA, and then realigned

81 to hg19 using the same parameters as the newly sequenced samples.

\section{Identification of somatic mutations in longitudinal samples}

84 Somatic mutations in the newly sequenced samples were identified using SAVI ${ }^{42}$. Briefly,

85 raw mutation calls as compared to the normal sample were generated from samtools 1.2

86 mpileup, and then annotated using SNPEff for their impacts ${ }^{43}$. SNPSIFT was used to add

87 further annotation such as whether the mutations are common SNPs (per dbSNP ${ }^{44}$ ), cancer

88 related somatic mutations (per COSMIC ${ }^{45}$ ), or observed in normal patients. A statistical

89 test was performed for each mutation call to determine the significance of being a somatic

90 mutation. SAVI is able to analyze mutations from multiple samples in one single run, which

91 is especially useful for longitudinal studies. 
92 For the published data, we identified somatic mutations using the same pipeline as the

93 newly sequenced ones when raw sequencing data were available. For the YALE, MSKCC

94 and GLASS cohort, we retrieved mutation call information (including mutation loci,

95 reference and alterative alleles, reference and alternative depth) from individual sources,

96 annotated with SAVI using the same parameters, and then applied the same filters to

97 identify somatic mutations. Mutations in known glioma driver genes were reported.

\section{Copy number alteration detection}

100 Copy number variations in the newly sequenced samples were detected using CNVkit $1010.9 .5^{46}$, which is suitable for both WES and WGS data. In both cases, only the exon regions 102 were considered. The BED file containing information about the captured regions was 103 downloaded from Agilent website (https://earray.chem.agilent.com/suredesign/index.htm).

104 The final results were segmented into SEG files.

105

\section{Detection of genes fusions, METex14 and EGFRvIII from RNA-seq data}

107 RNA sequencing data from previous publications were downloaded, and the reads were 108 extracted using samtools $1.2^{47}$. Starting from FASTQ files, STAR-fusion $1.5 .0^{48}$ was

109 utilized to identify and annotate gene fusion candidates, using the fastq files as input. The

110 fusion candidates were then filtered by removing fusions that were present in normal

111 tissues, fusions involving mitochondria genes and uncharacterized genes, and fusions of 112 two paralog genes. 
114 METex14 and EGFRvIII were identified by counting the spanning reads over the junction

115 of MET exon 13 and exon 15, and EGFR exon 1 and exon 8, respectively. Briefly, RNA

116 sequencing reads were aligned to the reference genome (hg19), and then the spanning reads

117 were extracted based on the CIGAR record. The spanning reads were manually checked in

118 Integrative Genomic Viewer to remove false positives such as PCR artifacts and potential

119 mapping errors. Only samples with at least three supporting reads were considered as 120 positive.

\section{Integration of mutation profiles}

123 For samples from the Yale, MSKCC and GLASS cohorts, all the mutations were obtained

124 from the original publications, which contains information about the coordinates of the

125 mutations, the reference and alternative alleles, as well as the read count of the reference

126 and alternative alleles. All the mutations were reannotated using SAVI, and the same

127 mutation filter as those used in the new samples were applied. This unified mutation

128 annotation and filtering method ensures the results from multiple cohorts comparable.

129 The copy number alteration calls from all samples were first transformed to SEG format

130 which included the coordinate of each segment and the estimated segment mean value

131 (reflecting the magnitude of copy number change). Subsequently, each segment was

132 annotated for the carried genes using the reference human genome GRCh37.75. The same

133 cutoffs for copy number gain, amplification and deletion were applied.

134 For gene fusions, where available, the raw sequencing reads were downloaded or extracted,

135 and then subjected to gene fusion detection using the same protocol. 


\section{Construction of Tumor Evolution Directed Graphs}

138 Tumor Evolution Directed Graphs ${ }^{49}$ were constructed using CELLO ${ }^{20}$. The input to

139 CELLO was the mutation calls from SAVI, and the samples were organized in the normal-

140 initial-recurrence order. CELLO automatically extracted the shared, private to initial and

141 private to recurrence mutations in each patient, which will be used to construct the tree.

142 The evolution trees were visualized in Cytoscape.

\section{Calculation of entropy and mutual information}

145 For each patient, an alteration might be gained or lost during evolution, both were counted

146 as one "status change event". If the alteration was present at both time points, or absent at

147 both time points, it is counted one "status stable event". For a given alteration X, suppose

148 the number of status change events in the cohort is $m$, and the number of "status stable

149 event" is $n$, then the entropy is

$150 \quad H(X)=-\frac{m}{m+n} \log _{2}\left(\frac{m}{m+n}\right)-\frac{n}{m+n} \log _{2}\left(\frac{n}{m+n}\right)$.

151 The mutual information between two alterations, $\mathrm{X}$ and $\mathrm{Y}$, is calculated as below:

$152 I(X, Y)=H(X)+H(Y)-H(X, Y)$.

153 In our analysis, the maximum number of features was set to four. All the possible

154 combinations were enumerated to find the four features with the highest mutual 155 information.

\section{Machine learning model training, validation, and feature importance}

158 XGBoost was used to train and test the models ${ }^{50}$. XGboost is a boosting machine learning 159 methodology, supporting influential supervised classification tasks. The dataset was 
160 divided into training set and test set two times for the prediction of HM and Grade with the

161 ratio of $3: 1$. For the training dataset, we leveraged five-fold cross validation to select the

162 optimal parameters, which were applied to the final configuration of XGBoost with best

163 validation AUC (Area Under receiver operating characteristic Curve). Then, the

164 independent test dataset was imported into the model to evaluate the performance and

165 generalization ability of the model for unseen data. The model training and testing was

166 implemented in R using the xgboost package, and SHAP (SHapley Additive exPlanations)

167 score $^{17}$ was extracted from the XGBoost models using SHAPforxgboost package.

168

169 Survival analysis

170 Survival analysis was performed using the survival package in $\mathrm{R}$. The significance levels

171 were calculated by two-sided log-rank test, and the Kaplan-Meier curves were plotted using

172 survminer package.

173

\section{Identification of hypermutation}

175 Hypermutation was detected using CELLO, with default parameters. CELLO determines

176 the hypermutation status based on mutation load and a hypermutation score which we

177 defined previously ${ }^{20}$. For the MSKCC cohort which includes panel sequencing data, the

178 mutation load reported in the publication was directed used, while the hypermutation score

179 was calculated using the same methodology as other samples.

180

181 Gene expression analysis 
182 Differential gene expression analysis was performed by the DEseq package 1.26.0. Gene

183 Set Enrichment Analysis was performed by the fgsea package (version 1.12.0) in R 3.6.3.

184 The gene sets regarding human cancer were gene sets which were downloaded from

185 MSigDB database version 6.1 (http://software.broadinstitute.org/gsea/msigdb). To

186 quantify global transcription activity, the mean expression level of 14 housekeeping genes:

187 ACTB, GAPDH, PGK1, PPIA, RPL13A, RPLP0, B2M, YWHAZ, SDHA, TFRC, GUSB,

188 HMBS, HPRT1 and TBP in each sample was calculated and the genes with expression

189 levels higher than this reference value was designated as "high-expression" genes.

191 ChIP-seq data acquisition and intersection with hypermutation loci

192 ChIP-seq data (listed in Table S3), including both binding density and binding peaks, were 193 downloaded from ChIP-Atlas (https://chip-atlas.org/). The peaks were extended by 1-

$1945,000,000$ bp upstream and downstream, and the overlaps, regions in the centromeres, 195 telomeres or out of chromosome borders were removed.

197 Recurrent-specific mutations from WGS data were divided into MYC-gained HM, non198 MYC-gained-HM and non-HM mutations, and randomly down-sampled to 150,000 for 199 each type. The sampling process was repeated for five times. The mutation sites were 200 transformed to BED format, and their intersection with the ChIP peaks were calculated 201 using bedtools v2.26.0. The mutation density was calculated by dividing the total number 202 of mutations with the total width of the regions. The mean and standard deviation of the 203 five replicates were calculated. 
206 The human glioma cell line U87 and U251were obtained from the Cell Resource Center,

207 Peking Union Medical College (Beijing, China), and the cell has been authenticated by the

208 short tandem repeat analysis Chronic temozolomide treatment cells. Both the two cells

209 were routinely cultured in Dulbecco's modified Eagle's medium (DMEM) supplemented

210 with $10 \%$ fetal bovine serum (HyClone, Logan, Utah), 100 units/ml penicillin and 100

$211 \mathrm{mg} / \mathrm{ml}$ streptomycin (Invitrogen, Carlsbad, CA) at $37^{\circ} \mathrm{C}$ in a humidified atmosphere of 5\%

$212 \mathrm{CO}_{2}$. The PDC T2-4, was obtained from fresh surgical specimens of a human primary GBM

213 and cultured as tumor spheres in DMEM/F12 medium supplemented with B27 supplement

214 (Life Technologies), bFGF and EGF (20 ng/ml each) at $37^{\circ} \mathrm{C}$ in a humidified atmosphere

215 of $5 \% \mathrm{CO}_{2}$.

217 Chronic temozolomide treatment of glioma cell lines

218 The human glioma cell line U87MG and U251MG were obtained from the Cell Resource

219 Center, Peking Union Medical College (Beijing, China). U87MG and U251MG cells were 220 treated with $\mathrm{TMZ}$ on Initial concentration of $100 \mu \mathrm{M}$ for $72 \mathrm{~h}$. TMZ-sensitive cells

221 generated death, and the survived cells are collected for further resistant induction with 222 increasing TMZ concentration $(\leq 200 \mu \mathrm{M})$. After more than 20 generations and 6 months

223 later, each cell line's cells survived and growth in DMEM culture medium containing TMZ

224 of final concentration of $200 \mu \mathrm{M}$, were referred as U87TR and U251TR cells.

225 The PDCs were treated with TMZ on an initial concentration of $50 \mu \mathrm{M}$ for 5 days, and the

226 remain cells were incubated in medium with increasing concentration of TMZ until to 200

$227 \mu \mathrm{M}$. The IC50 values of TMZ were determined in the following situations: 1) after 6 
228 generations of growth in medium with $200 \mu \mathrm{M} \mathrm{TMZ}$; 2) the PDCs reached the status that

229 they have similar growth rates in normal medium and medium containing $200 \mu \mathrm{M} \mathrm{TMZ}$.

230 Finally, the PDCs were considered TMZ-resistant when they have significantly elevated

231 IC50 values compared with their corresponding initial cells.

233 Temozolomide sensitivity test

234 The temozolomide sensitivity were studied by CCK-8 kit (Dojindo Laboratories,

235 Kumamoto, Japan) according to the manufacturer's protocol. 3000 cells were grown in 236 each well of $96-w e l l$ plates with normal medium for $24 \mathrm{hr}$. Then the cells were incubated at

237 medium containing TMZ at predesigned concentrations for $72 \mathrm{hrs}$, and there were six238 repeated wells for each predesigned concentrations. Next, $10 \mu \mathrm{CCK}-8$ reagent was added

239 to the medium of each well, and the absorbances at $450 \mathrm{~nm}$ and $630 \mathrm{~nm}$ of the medium

240 were measured after incubation for 2 hours. The number of living cells could be reflected

241 by the difference between optical density of $450\left(\mathrm{OD}_{450}\right)$ and $630\left(\mathrm{OD}_{630}\right) \mathrm{nm}$ wavelength.

242 The response curves were fitted using the $d r c$ package 3.0-1.

\section{Western blot}

245 The whole-cell lysates were using RIPA buffer [150 mM NaCl, 0.1\% (wt/vol) NP-40, 50

$246 \mathrm{mM}$ Tris $(\mathrm{pH} 8.0), 0.5 \%(\mathrm{wt} / \mathrm{vol})$ sodium deoxycholate, $1 \%(\mathrm{wt} / \mathrm{vol})$ sodium dodecyl sulfate,

$2471 \mathrm{mM}$ dithiothreitol, $0.1 \mathrm{mM}$ phenylmethylsulfonyl fluoride]. A bicinchoninic acid (BCA)

248 array was used to measure the total protein content. The equal total proteins $(20 \mu \mathrm{g})$ were

249 boiled and then were electrophoresed on a $8 \%$ and $12 \%$ sodium dodecyl sulfate-

250 polyacrylamide gel electrophoresis gel, respectively. After membrane transfer and 
251 blocking, the primary antibodies and used to probe the target proteins in blocking solution

252 overnight at $4^{\circ} \mathrm{C}$. The membrane were detected using an ECL Western Blotting Detection

253 System (Bio-Rad) after Goat anti-rabbit IgG-HRP or goat anti-mouse IgG-HRP incubation

$2541 \mathrm{hr}$ at room temperature. Glyceraldehyde 3-phosphate dehydrogenase (GAPDH) was used

255 as the loading control.

256

257 Generation of lentiviral vectors encoding c-MYC

258 The sequencing of c-MYC (NM_002467) was cloned from glioma samples. The sequence

259 was inserted into the lentiviral vector plasmid GV358 (Genechem). The GV358 plasmid

260 carrying the c-MYC CDS sequencing was transduced into 293T cells with the lentiviral

261 packaging plasmid mix (Genechem). The culture medium was collected once a day on two

262 consecutive days and centrifuged with an ultracentrifuge at $25,000 \mathrm{rpm}$ for $1.5 \mathrm{~h}$. The

263 precipitate was resuspended, aliquoted and stored at $-80^{\circ} \mathrm{C}$. This preparation was added to

264 the U87 cell culture or PDCs supplemented with $3 \mathrm{mg} / \mathrm{ml}$ polybrene (Sigma) in the medium.

265 The medium was changed after $24 \mathrm{~h}$. After $72 \mathrm{~h}$, cells containing vectors were selected

266 during 3 days of treatment with puromycin. The c-MYC expression in the U87 cells and

267 PDCs were confirmed by Western blot.

268

269 Validation of MSH6 mutation by Sanger sequencing

270 DNA extraction and PCR were performed to get the fragment including the MHS6 271 mutation site identified by the whole exon sequencing, the primers sequences were as 272 follows: forward 5'-CTCCCCATGGGCTGCTAAG-3', Reverse 5' 273 TATGTCCTAGGCGCACAGC-3'. The product bands were extracted from agarose gel 274 after electrophoresis and verified by Sanger sequencing with the forward primer. 


\section{Code availability}

277 The hypermutation status, and the Tumor Evolution Directed Graphs were determined

278 using CELLO which is available at https://github.com/WangLabHKUST/CELLO. All

279 other scripts are available from the authors upon reasonable request.

\section{Data availability}

282 The processed genomic and transcriptomic data are available in the CELLO2 website

283 (https://wanglab.shinyapps.io/cello). Raw sequencing data of the newly sequenced samples

284 will be deposited to European Genome-Phenome Archive (EGA, https://ega-archive.org/).

285 Data from previous SMC samples were available in EGA 286 (https://www.ebi.ac.uk/ega/datasets/EGAS00001001800), data from TCGA were

287 downloaded from NCI Genomics Data Commons (GDC) data portal 288 (https://portal.gdc.cancer.gov). Previously published CGGA data have been uploaded to 289 the Genome Sequence Archive in BIG Data Center, Beijing Institute of Genomics (BIG),

290 Chinese Academy of Sciences, under accession number BioProject ID: PRJCA001636

291 (https://bigd.big.ac.cn/bioproject/browse/PRJCA001636) and PRJCA001747

292 (https://bigd.big.ac.cn/bioproject/browse/PRJCA001747). All the other data supporting the

293 findings of this study are available within the article and its information files and from the 294 corresponding author upon reasonable request. 


\section{Additional information}

297 Supplementary Information is available for this paper. Correspondence and requests for 298 materials should be addressed to Jiguang Wang (jgwang@ust.hk) or Tao Jiang 299 (taojiang1964@163.com).

300 Supplementary item titles and legends

301 Supplementary Table S1: Clinical characteristics of the longitudinal glioma cohort, 302 related to Figure 1.

303 Supplementary Table S2: Sequencing platforms used for the samples in the longitudinal 304 glioma cohort, related to Figure 1.

305 Supplementary Table S3: List of ChIP-seq data used in hypermutation distribution 306 analysis, related to Figure 4. 


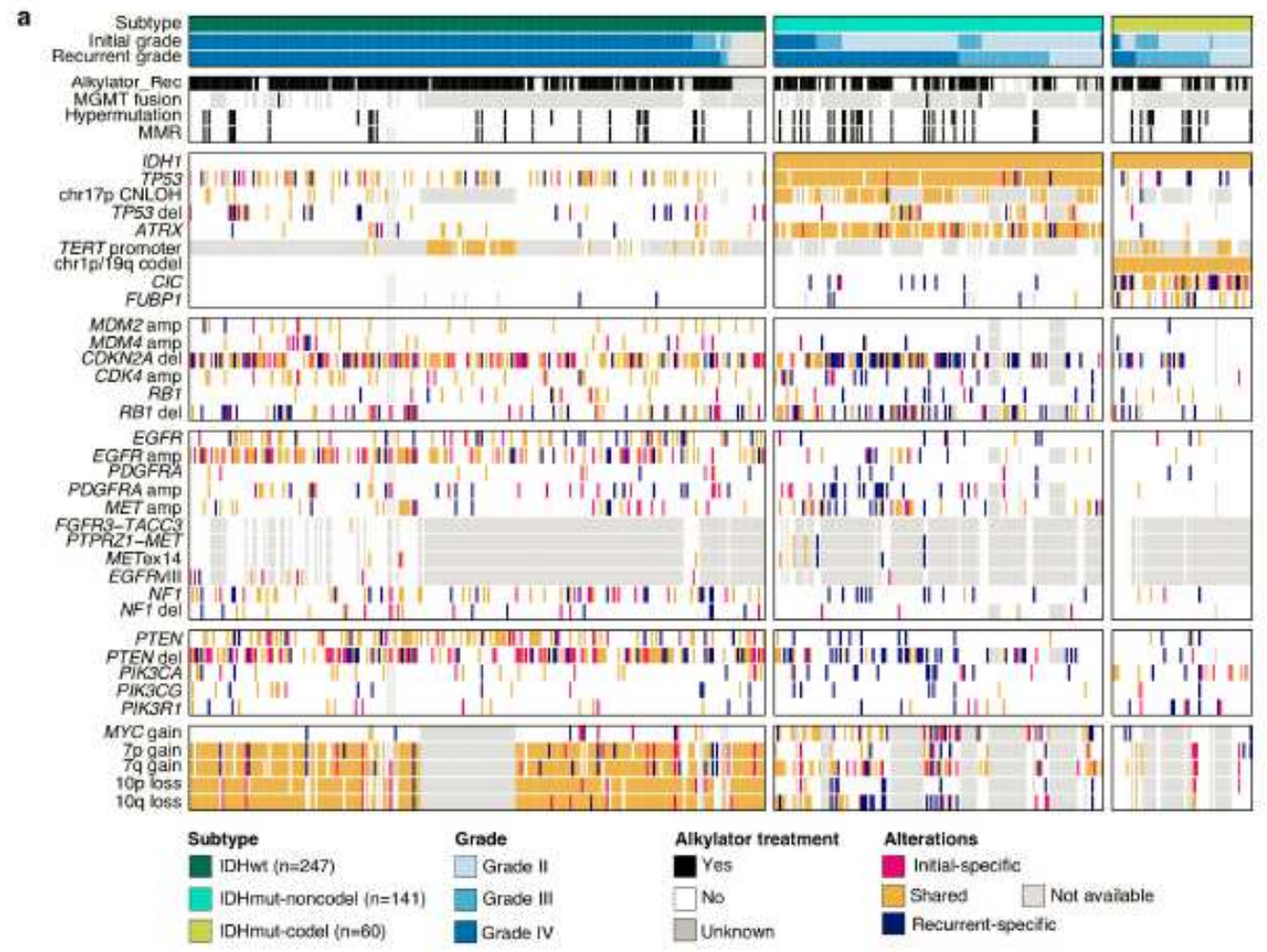

b
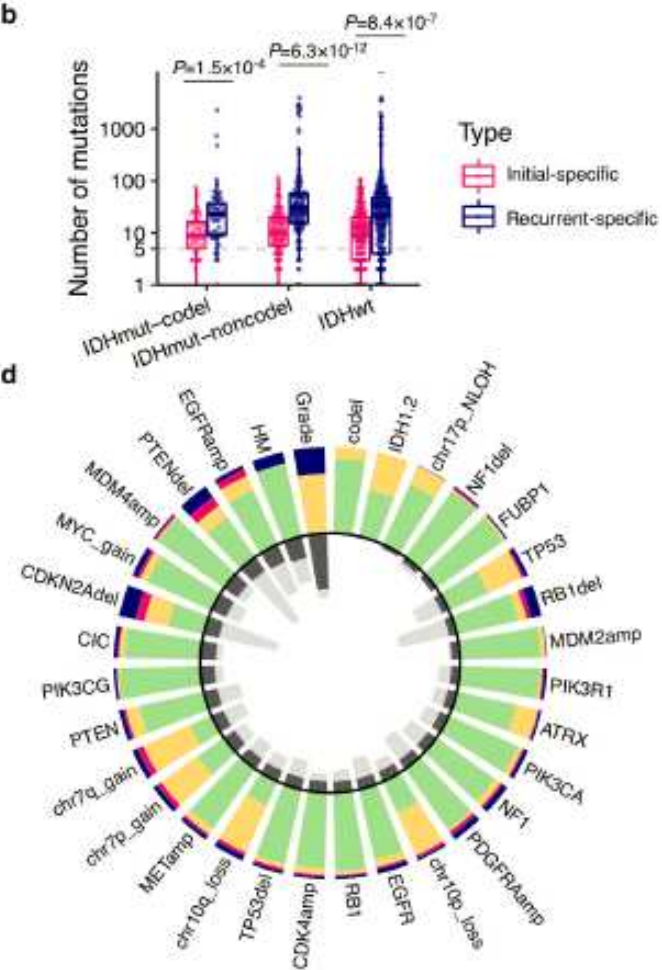

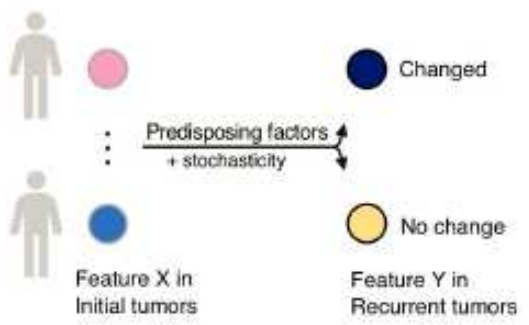

\section{Figure 1}

Identification of predictable events from pan-glioma evolutionary landscape. (a) Longitudinal molecular landscape of glioma patients with paired DNA sequencing data. Each column represents a patient which were stratified by the molecular subtype, and each row represents a clinical feature or molecular variant. 
The color of each variant indicates whether it is specific to initial, recurrence or shared. MMR: mismatch repair; CNLOH: copy-neutral loss of heterozygosity. (b) Comparison of the number of initial-and recurrent specific somatic mutations during evolution in each glioma subtype. The horizontal dashed line represents five mutations. The $\mathrm{P}$ values were calculated by Wilcoxon's rank-sum test. (c) Proposed model explaining the changes of the clinical and molecular features over time. Predisposing factors in the initial tumor, together with stochasticity, determine whether the status of the feature will change at recurrence. (d) Alteration status, entropy and mutual information of clinical and genomic features. Stacked bars outside the circle summarizes the frequency of a clinical feature or molecular variant to be lost, gain, remaining wildtype or remaining altered. Inside the circle shows the overlapped bar plots of mutual information (black) and conditional entropy (grey), where high proportion of overlap indicates better predictability. The features are ranked by mutual information. 
a

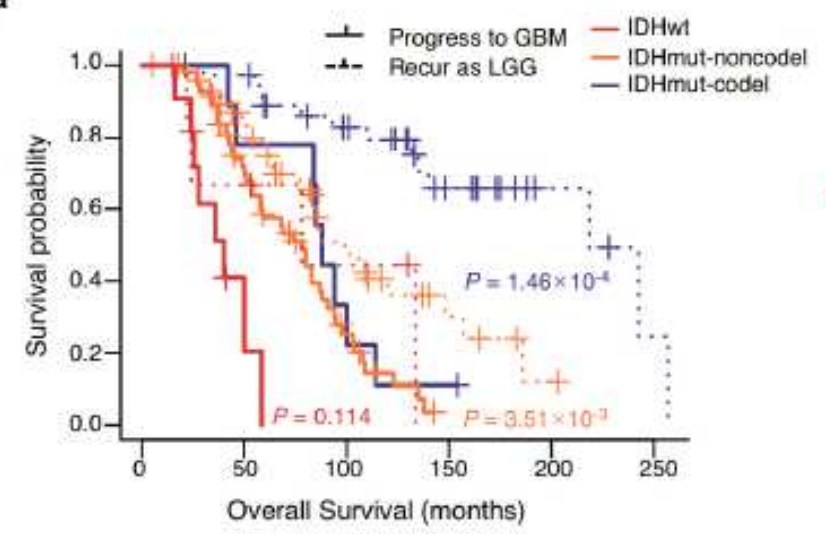

b

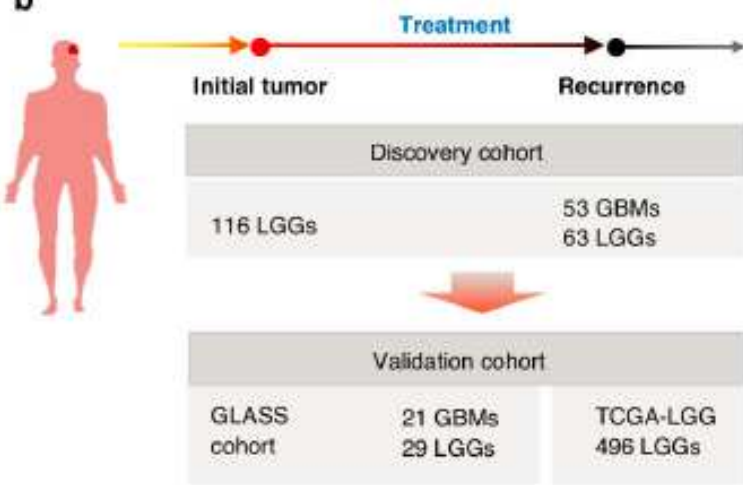

c

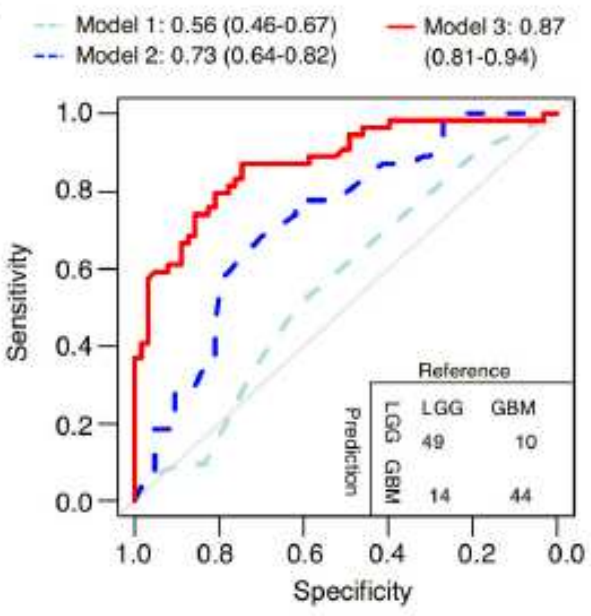

e
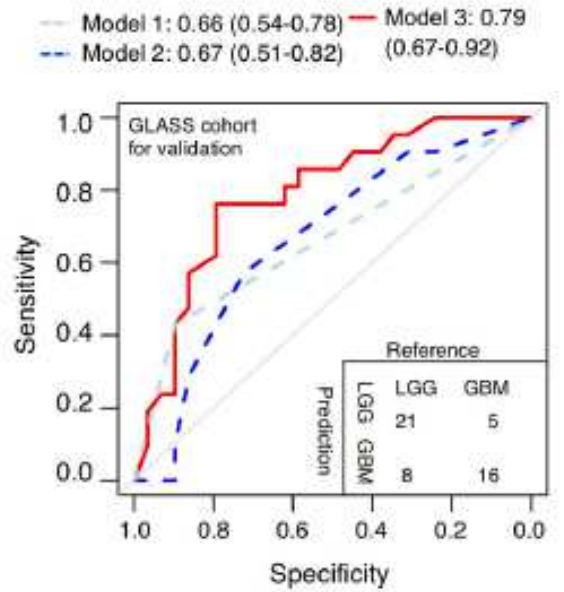

d

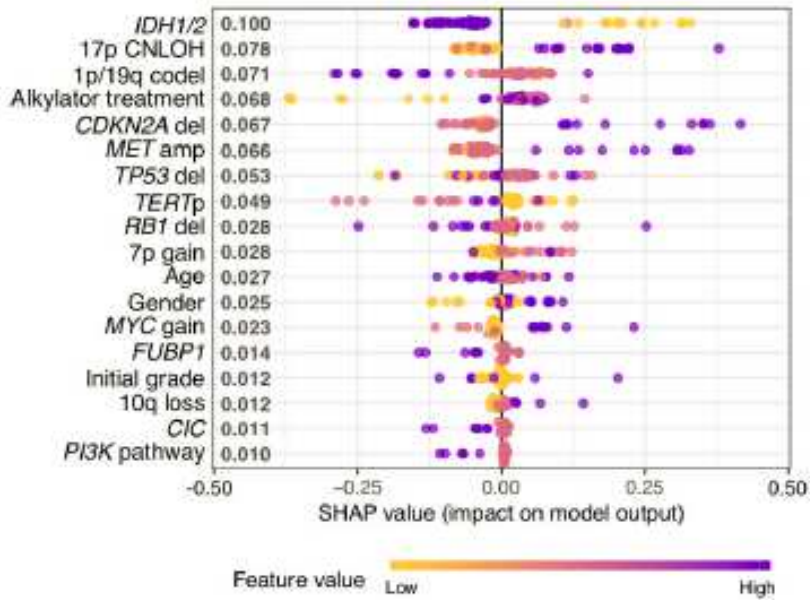

f

1 Predicted to progress $\perp$ Predicted to recur to $\mathrm{GBM}(\mathrm{n}=192$ )

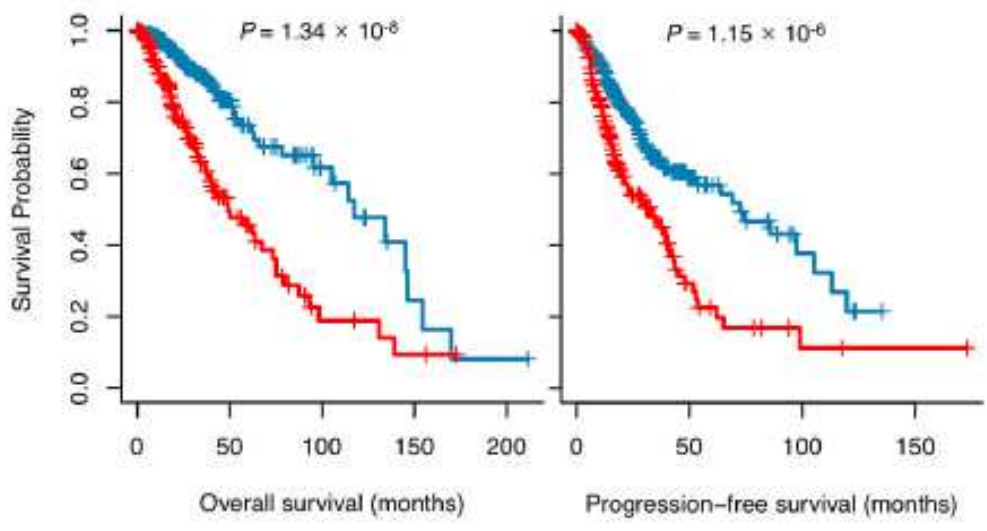

\section{Figure 2}

Genomic characteristics of initial glioma informs grade progression after treatment. (a) Overall survival of low-grade gliomas that progressed to glioblastoma (GBM) and those that recurred as low-grade glioma (LGG). The colors represent glioma subtypes, while the line type (solid or dashed) show whether the patient progressed to GBM at recurrence. The P values were calculated by log-rank test. (b) Design of the machine learning tasks. The discovery cohort, including 117 LGGs, was used to train machine learning 
models to predict whether the initial LGG patients would progress to GBM at recurrence. The models were then applied to two independent cohorts for validation. (c) Receiving operation characteristic (ROC) curves showing the cross-validation performance of the models trained using different combinations of features. Model 1: the initial grade as the single predictor; Model 2: the initial grade, age, gender, and treatment as predictors; Model 3: the initial grade, age, gender, treatment, and genomic alterations as predictors. (d) Shapley additive explanation (SHAP) scores of features in the prediction model of grade progression. The color of the points represents value of each feature. (e) ROCs showing the three models' prediction performance in the GLASS cohort. A confusion matrix is shown at the right bottom. (f) Overall survival (left panel) and progression-free survival (right panel) of LGGs from TCGA-LGG cohort, stratified by their predicted risk of progressing to GBM. Patients that were in this longitudinal cohort were excluded. The $P$ values were calculated by log-rank test. 
a

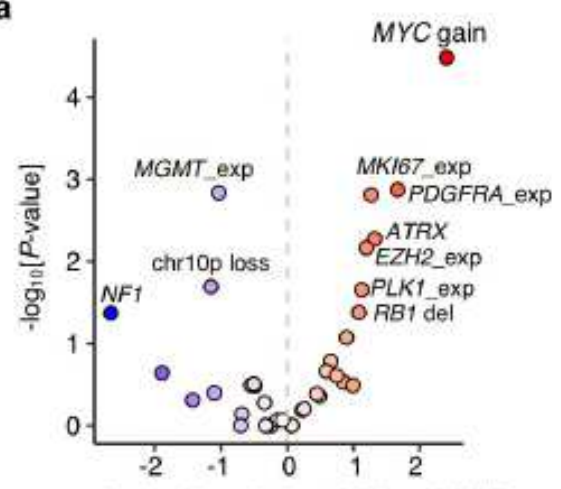

$\log _{2}[$ odds ratio (pre-HM/pre-NHM)]

d

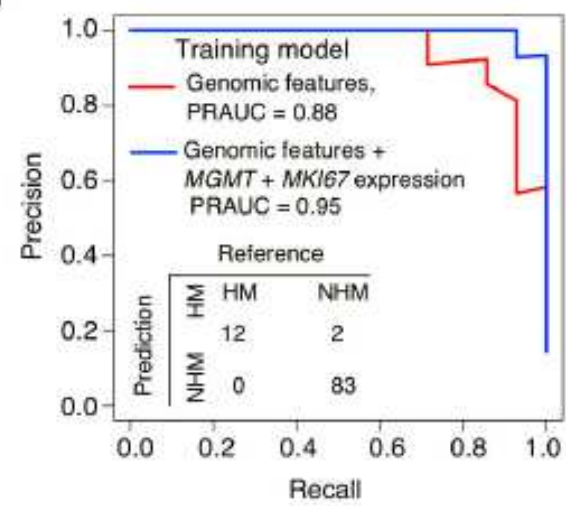

f
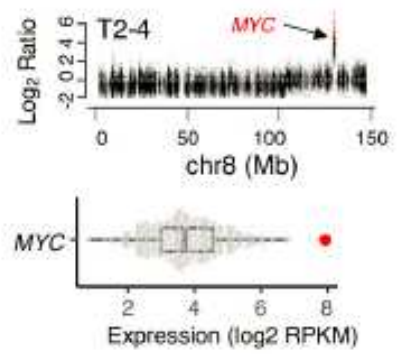

i
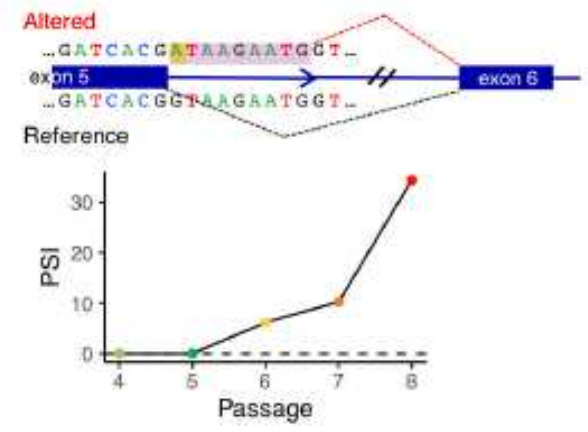

g b

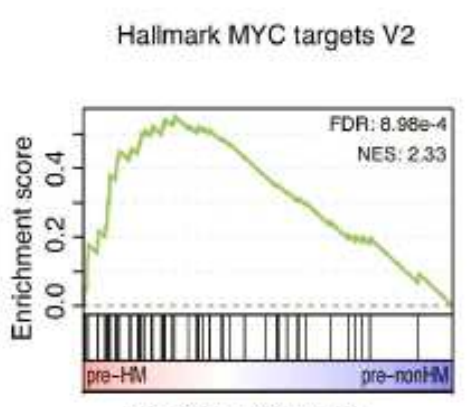

Rank in ordered list
C

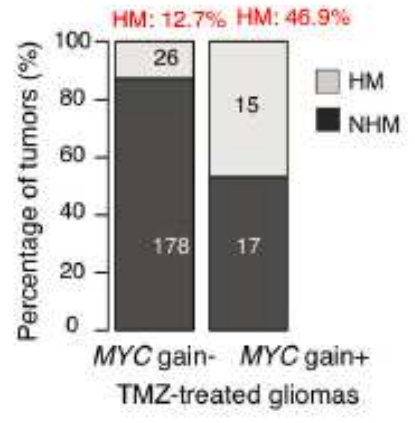

e

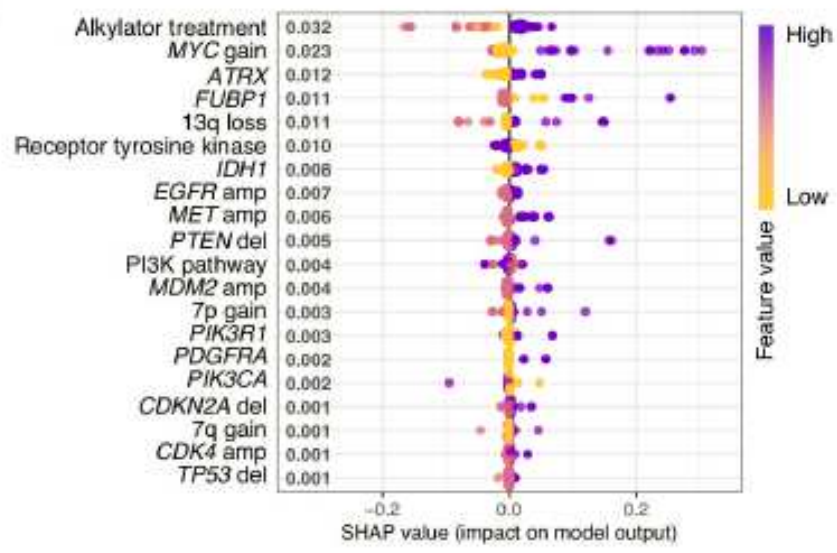

h
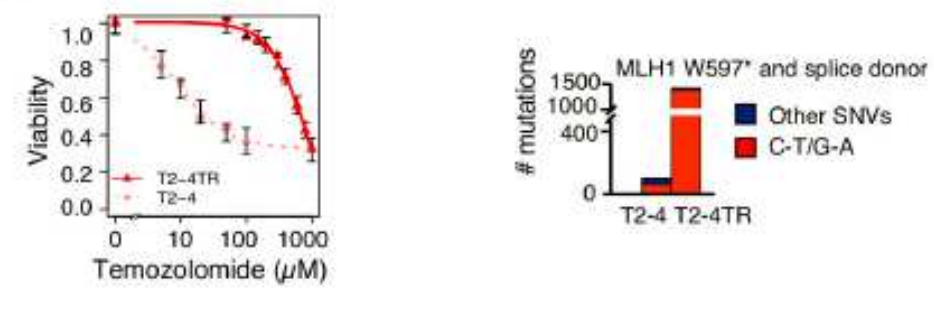

j
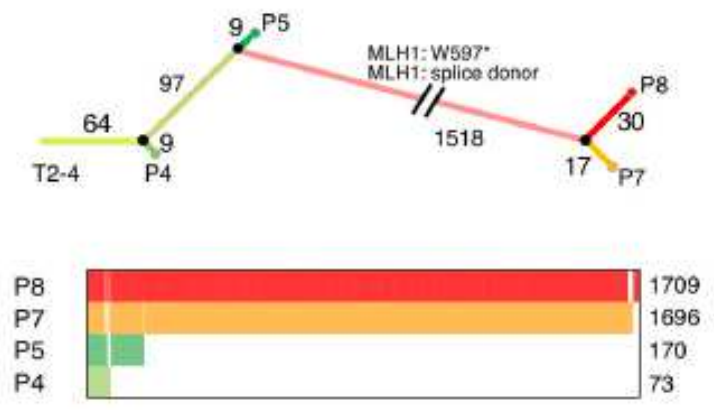

\section{Figure 3}

Features of initial tumors predispose TMZ-associated hypermutation at recurrence. (a) Comparison of genomic and transcriptomic features of the TMZ-treated initial gliomas preceding hypermutators (preHM) and initial tumors preceding non-hypermutators (pre- NHM). (b) Gene set enrichment analysis. Gene expression profile of pre-HM gliomas were compared to pre-NHM gliomas. FDR: false-discovery rate; NES: normalized enrichment score. (c) The frequency of developing hypermutation in TMZ treated gliomas 
with or without MYC copy number gain. (d) Precision-recall curve of HM prediction the model trained using genomic features (red curve) and genomic features plus expression of MGMT and MKI67 (blue curve). (e) SHAP value of each feature in the model trained with genomic features plus expression of MGMT and MKI67 in (c). (f) The DNA copy number of MYC in T2-4 (upper panel), and the expression level (lower panel) of T2-4 (the red dot) and other RNA sequenced gliomas in this study (grey dots). (g) Response curves of T2-4 and the induced T2-4TR cell line to TMZ. (h) Number of somatic mutations in T2-4 and T2-4 TR. Dark red represents $\mathrm{C}$ to $\mathrm{T}$ or $\mathrm{G}$ to $A$ mutations, while dark blue represents other somatic mutations. (i) Aberrant splicing related to the MLH1 splicing donor mutation in T2-4. The upper panel shows the aberrant splicing which included nine additional base pairs in the spliced mRNA. The lower panel shows the percentage of spliced-in (PSI) reads of the aberrant isoform in different passages of T2-4. (j) Mutations in T2-4 at different time points during the TMZ-inducing experiment. The upper panel shows the phylogenetic tree constructed from the mutations in passages 4, 5, 7 and 8 as compared to the untreated T2- 4 PDC, and the lower panel shows a heatmap of the mutations. 
a

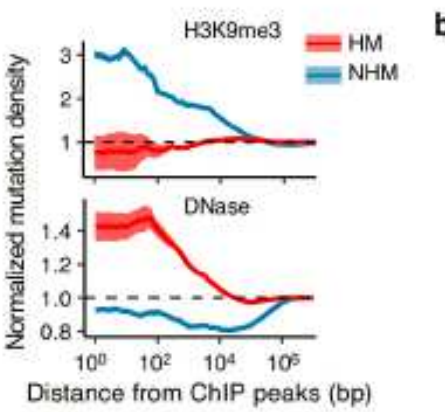

b

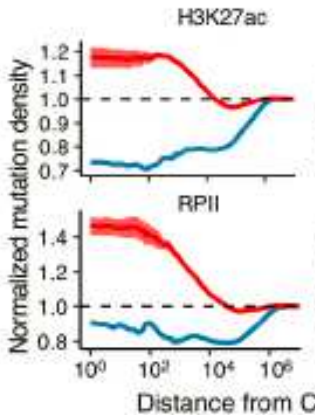

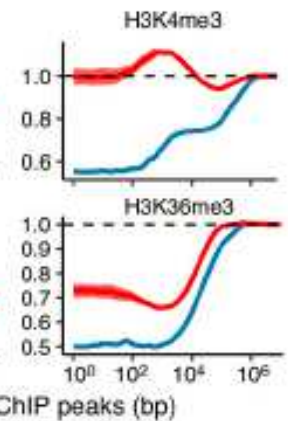

C

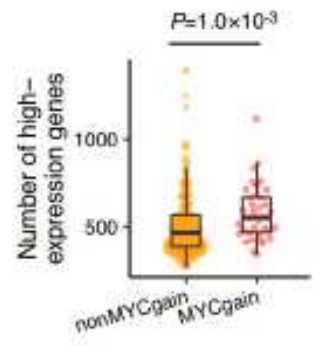

d

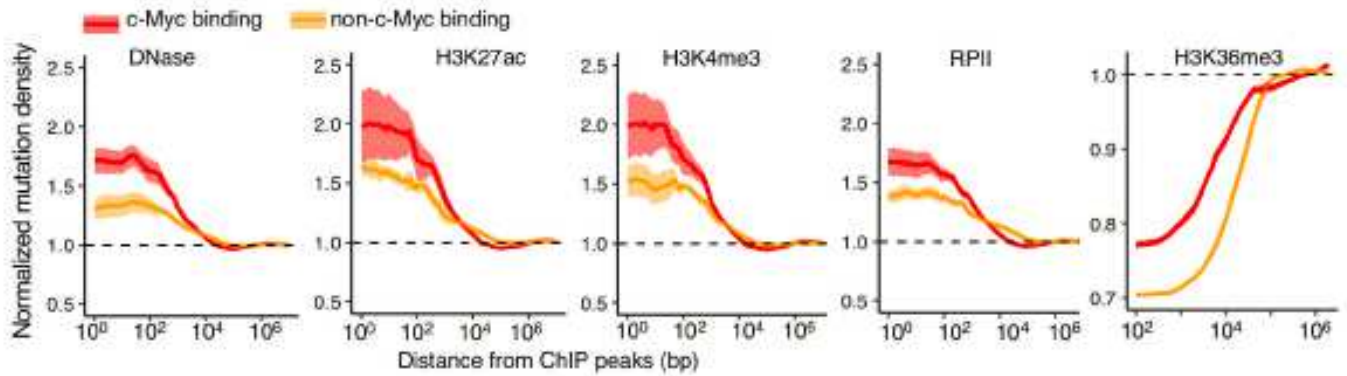

e
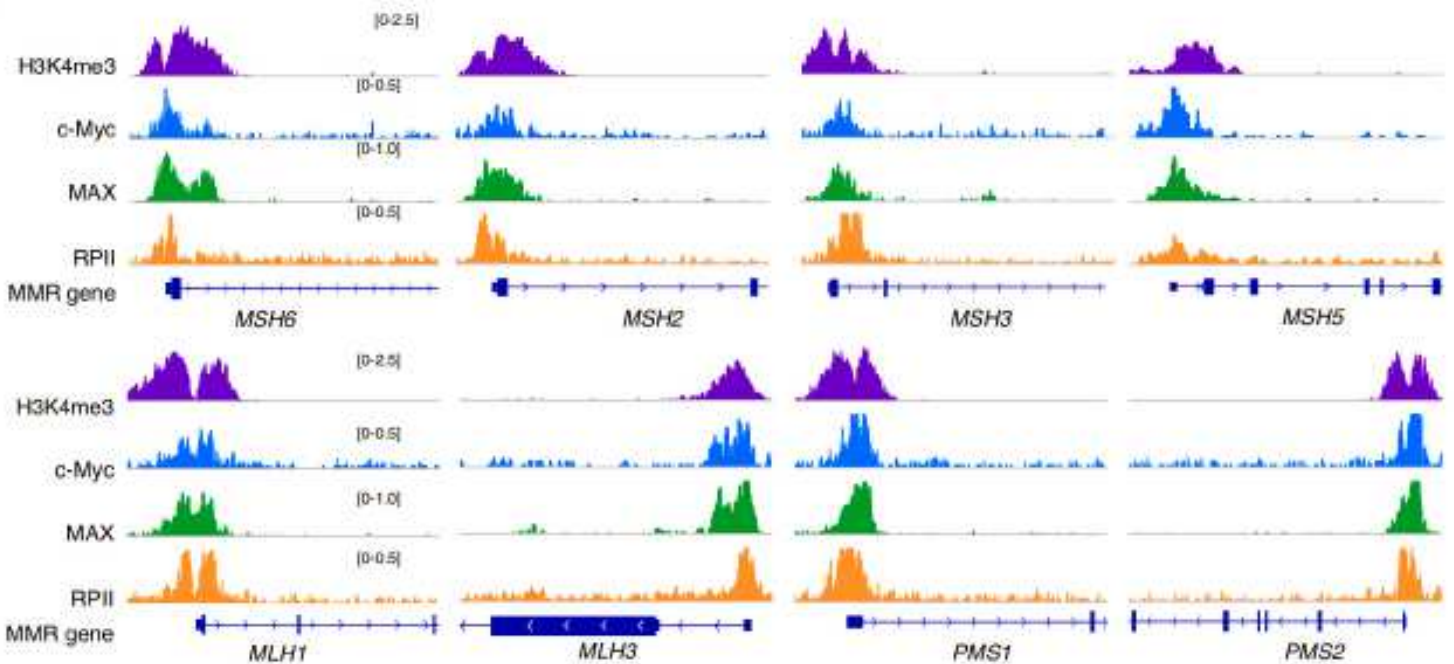

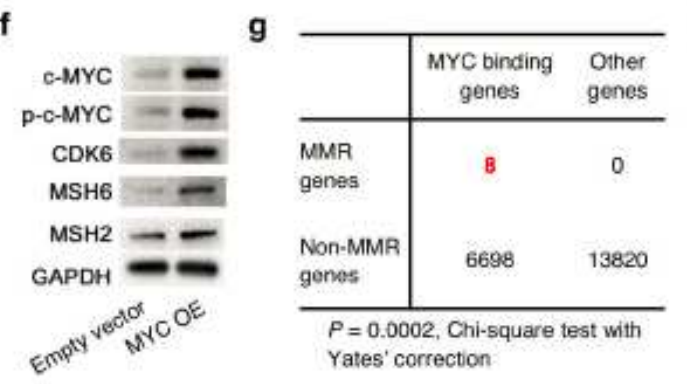

h

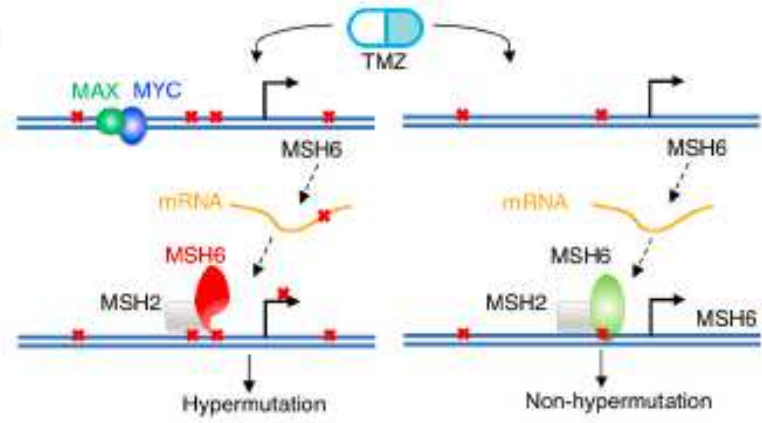

Figure 4

TMZ-induced mutations are enriched in open active regions, while c-Myc binding further increases mutation density (a) Normalized mutation density of TMZ-associated hypermutations (HM) and nonTMZ associated recurrence-specific somatic mutations (NHM) around H3K9me3 modification sites (upper panel) and DNase hypersensitive sites (lower panel). The dashed horizontal line represents the random level. (b) Normalized mutation density of HM and NHM around H3K27ac modification sites 
(upper left), H3K4me3 modification sites (upper right), RNA polymerase II (RPII) binding sites (lower left) and H3K36me3 modification sites (lower right). (c) Number of high-expression genes in gliomas with and without MYC gain. The P value was calculated by Wilcoxon's rank-sum test. (d) Normalized mutation density of HM around DNase hypersensitive sites, H3K27ac modification sites, H3K4me3 modification sites, RPII binding sites and H3K36me3 modification sites with (red color) and without (orange color) cMyc binding. (e) Chromatin immunoprecipitation (ChIP) intensity of H3K4me3 modification, c-Myc, MAX and RPII binding at eight mismatch repair (MMR) genes. (f) Contingent table of c-Myc binding and MMR genes. (g) Western blot showing c-MYC, p-c-MYC, CDK6, MSH6, MSH2, and MLH1 in U87 cell lines with empty vector or MYC overexpression. OE: overexpression. (h) Proposed working model of how MYC activation increases chance of hypermutation.

\section{Supplementary Files}

This is a list of supplementary files associated with this preprint. Click to download.

- TableS1.Patient.Clinicallnformation.xlsx

- TableS2.Sequencing.Data.xlsx

- SupplementaryInformationDec30.docx 